\title{
Identification of "Multiple Components-Multiple Targets-Multiple Pathways" Associated with Naoxintong Capsule in the Treatment of Heart Diseases Using UPLC/Q-TOF-MS and Network Pharmacology
}

\author{
Xianghui Ma, ${ }^{1}$ Bin Lv, ${ }^{1}$ Pan Li, ${ }^{1}$ Xiaoqing Jiang, ${ }^{1}$ Qian Zhou, \\ Xiaoying Wang, ${ }^{1,2}$ and Xiumei Gao ${ }^{1}$ \\ ${ }^{1}$ State Key Laboratory of Modern Chinese Medicine, Tianjin University of Traditional Chinese Medicine, Tianjin 300193, China \\ ${ }^{2}$ College of Traditional Chinese Medicine, Tianjin University of Traditional Chinese Medicine, Tianjin 300193, China
}

Correspondence should be addressed to Xiaoying Wang; wxy@tjutcm.edu.cn

Received 30 January 2016; Accepted 2 March 2016

Academic Editor: Carmen Mannucci

Copyright (C) 2016 Xianghui Ma et al. This is an open access article distributed under the Creative Commons Attribution License, which permits unrestricted use, distribution, and reproduction in any medium, provided the original work is properly cited.

\begin{abstract}
Naoxintong capsule (NXT) is a commercial medicinal product approved by the China Food and Drug Administration which is used in the treatment of stroke and coronary heart disease. However, the research on the composition and mechanism of NXT is still lacking. Our research aimed to identify the absorbable components, potential targets, and associated pathways of NXT with network pharmacology method. We explored the chemical compositions of NXT based on UPLC/Q-TOF-MS. Then, we used the five principles of drug absorption to identify absorbable ingredients. The databases of PharmMapper, Universal Protein, and the Molecule Annotation System were used to predict the main targets and related pathways. By the five principles of drug absorption as a judgment rule, we identified 63 compositions that could be absorbed in the blood in all 81 chemical compositions. Based on the constructed networks by the significant regulated 123 targets and 77 pathways, the main components that mediated the efficacy of NXT were organic acids, saponins, and tanshinones. Radix Astragali was the critical herbal medicine in NXT, which contained more active components than other herbs and regulated more targets and pathways. Our results showed that NXT had a therapeutic effect on heart diseases through the pattern "multiple components-multiple targets-multiple pathways."
\end{abstract}

\section{Introduction}

Naoxintong capsule (NXT) is a commercial medicinal product approved by the China Food and Drug Administration which is widely used in the treatment of stroke and coronary heart disease. NXT contains 16 Chinese herbal medicines (Table 1). NXT exerts significant therapeutic effects and has high safety for stroke recovery in the clinical setting [1]. Recent studies showed that NXT could reduce the infarct size of acute myocardial infarction (AMI) patients by improving vascular endothelial function [2]. Long-term administration of NXT was also reported to alleviate inflammation, reduce the recurrence of angina pectoris, and decrease the incidence of ACS attack in borderline lesion coronary heart disease patients [3]. Some studies investigated the mechanisms of
NXT in vitro or in vivo. NXT was reported to protect against atherosclerosis through its lipid-lowering activity [4] and to reduce the expression of iNOS mRNA and the NO level in the vessel wall to benefit the treatment of atherosclerosis [5]. NXT also protected cardiomyoblasts against $\mathrm{H}_{2} \mathrm{O}_{2}$ induced oxidative injury [6]. Although some mechanisms of NXT have been reported, existing studies on unilateral factors and single targets could not demonstrate the complex mechanisms of NXT, a herbal prescription with 16 medicines which is prescribed for the treatment of complex diseases like cardiovascular and cerebrovascular diseases.

With the prominence of network pharmacology in system biology, this distinct and novel approach to the study of complicated analytical systems is becoming more widely known and more frequently used in the field of drug research. 
TABLE 1: Sixteen Chinese traditional medical herbs of NXT.

\begin{tabular}{|c|c|c|c|}
\hline Abbreviation & Medicinal herbs & Original plants & Content $(\mathrm{g})^{*}$ \\
\hline RA & Radix Astragali & $\begin{array}{l}\text { Astragalus membranaceus (Fisch.) Beg. var. mongholicus } \\
\text { (Bge.) Hsiao or A. membranaceus (Fisch.) Bge. }\end{array}$ & 66 \\
\hline RPR & Radix Paeoniae Rubra & Paeonia lactiflora Pall. or P. veitchii Lynch & 27 \\
\hline RSM & Radix Salviae Miltiorrhizae & Salvia miltiorrhiza Bge. & 27 \\
\hline RAS & Radix Angelicae Sinensis & Angelica sinensis (Oliv) Diels. & 27 \\
\hline $\mathrm{RCX}$ & Rhizoma Chuanxiong & Ligusticum chuanxiong Hort. & 27 \\
\hline SP & Semen Persicae & $\begin{array}{l}\text { Prunus persica (L.) Batsch or Prunusdavidiana (Carr.) } \\
\text { Franch. }\end{array}$ & 27 \\
\hline FC & Flos Carthami & Carthamus tinctorius L. & 13 \\
\hline FK & Frankincense & Boswellia carterii Birdw. & 13 \\
\hline $\mathrm{MRH}$ & Myrrha & Commiphora myrrha Engl. & 13 \\
\hline CS & Caulis Spatholobi & Spatholobus suberectus Dunn & 20 \\
\hline RAB & Radix Achyranthis Bidentatae & Achyranthes bidentata Bl. or Cyathula officinalis Kuan & 27 \\
\hline $\mathrm{RC}$ & Ramulus Cinnamomi & Cinnamomum cassia Presl & 20 \\
\hline $\mathrm{RM}$ & Ramulus Mori & Morus alba L. & 27 \\
\hline PT & Pheretima & $\begin{array}{l}\text { Pheretima aspergillum (E. Perrier) or Pheretima vulgaris } \\
\text { Chen. or Pheretima guillelmi (Michaelsen) or Pheretima } \\
\text { pectinifera Michaelsen }\end{array}$ & 27 \\
\hline SCP & Scorpio & Buthus martensii Karsch & 13 \\
\hline HRD & Hirudo & $\begin{array}{l}\text { Whitmania pigra Whitman or Hirudo nipponica } \\
\text { Whitman or Whitmania acranulata Whitman }\end{array}$ & 27 \\
\hline
\end{tabular}

* Note. The content of 16 Chinese traditional medical herbs of NXT came from Chinese Pharmacopoeia 2015.

The functions of network pharmacology include uncovering the functions of traditional Chinese medicines (TCMs), providing deeper insights into and scientific evidence for TCMs, and identifying TCMs as scientifically proven. Here, we attempt to explore the mechanism of NXT using this method.

In the current study, based on the use of UPLC/QTOF-MS to investigate the involved components, we aimed to analyse the absorbable components of NXT, to identify potential targets and associated pathways using the network pharmacology method, and to systematically discuss the mechanism of NXT in the treatment of heart diseases.

\section{Material and Methods}

\subsection{Prediction of Components}

2.1.1. Sample Preparation. NXT was obtained from HezeBuchang Pharmaceutical Co., Ltd. (Heze, China). Deionized water was prepared from aqua distillate using a Milli-Q system (Millipore, Bedford, MA, USA). Analytical grade methanol was purchased from Merck (Darmstadt, Germany). We dissolved $1 \mathrm{~g}$ of NXT powder in $10 \mathrm{~mL}$ of $75 \%$ analytical grade methanol and subjected the mixture to ultrasonic extraction for $30 \mathrm{~min}$. We then brought the solution to room temperature and obtained the supernatant as a capture reagent. The sample was filtered using a $0.22 \mu \mathrm{m}$ microporous membrane before UPLC analysis.
2.1.2. UPLC/Q-TOF-MS. We used a Waters Acquity UPLC System (Waters Co., USA) furnished with a photodiode array detector for the analysis. The sample was diluted on a Waters Acquity UPLC BEH C18 column $(2.1 \mathrm{~mm} \times$ $100 \mathrm{~mm}, 1.7 \mu \mathrm{m})$. UV detection was achieved at 190-400 nm. The system was controlled using the MassLynx version 4.1 software (Waters Co.). The gradient duration program for A (UPLC-grade acetonitrile) and B (water with $0.1 \%$ formic acid) was performed as follows: $2 \% \mathrm{~A}$ from $0 \mathrm{~min}$ to $3 \mathrm{~min}$, $10 \%$ to $50 \%$ A from $3 \mathrm{~min}$ to $12 \mathrm{~min}, 50 \%$ to $63 \%$ A from $12 \mathrm{~min}$ to $18 \mathrm{~min}, 63 \%$ to $83 \%$ A from $18 \mathrm{~min}$ to $21 \mathrm{~min}, 83 \%$ to $84 \%$ A from $21 \mathrm{~min}$ to $22 \mathrm{~min}, 84 \%$ to $87 \%$ A from $22 \mathrm{~min}$ to $26 \mathrm{~min}, 87 \%$ to $90 \%$ A from $26 \mathrm{~min}$ to $28 \mathrm{~min}, 90 \%$ to $95 \%$ A from $28 \mathrm{~min}$ to $31 \mathrm{~min}, 95 \%$ to $100 \%$ A from 31 to $33 \mathrm{~min}, 100 \%$ to $100 \%$ A from 33 to $35 \mathrm{~min}$, and $100 \%$ to $2 \%$ A from $35 \mathrm{~min}$ to $37 \mathrm{~min}$. The flow rate was maintained at $0.4 \mathrm{~mL} / \mathrm{min}$, and the column temperature was maintained at $30^{\circ} \mathrm{C}$.

The components of NXT were identified using a Waters Q-TOF Premier with an electrospray ionization (ESI) system (Waters MS Technologies, Manchester, UK). The ESI-MS spectra were acquired at both negative and positive ion voltages. The capillary voltage was set to $2.5 \mathrm{kV}$ for the negative mode and to $3.0 \mathrm{kV}$ for the positive mode. The sample cone voltage was set to $30 \mathrm{~V}$, and the source temperature was $110^{\circ} \mathrm{C}$. High-purity nitrogen was used as the nebulization and auxiliary gas. The nebulization gas was set to $600 \mathrm{~L} / \mathrm{h}$, the cone gas was set to $50 \mathrm{~L} / \mathrm{h}$, and the desolation temperature was $350^{\circ} \mathrm{C}$. The Q-TOF Premier acquisition rate was $0.1 \mathrm{~s}$, and there was a $0.02 \mathrm{~s}$ interscan delay. Argon, which was 


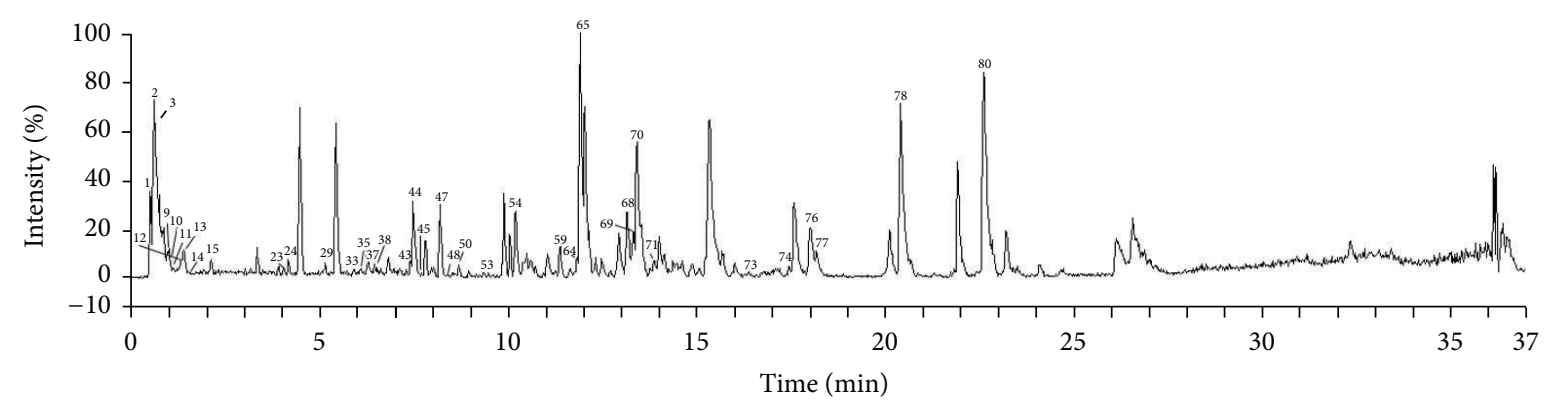

(a)

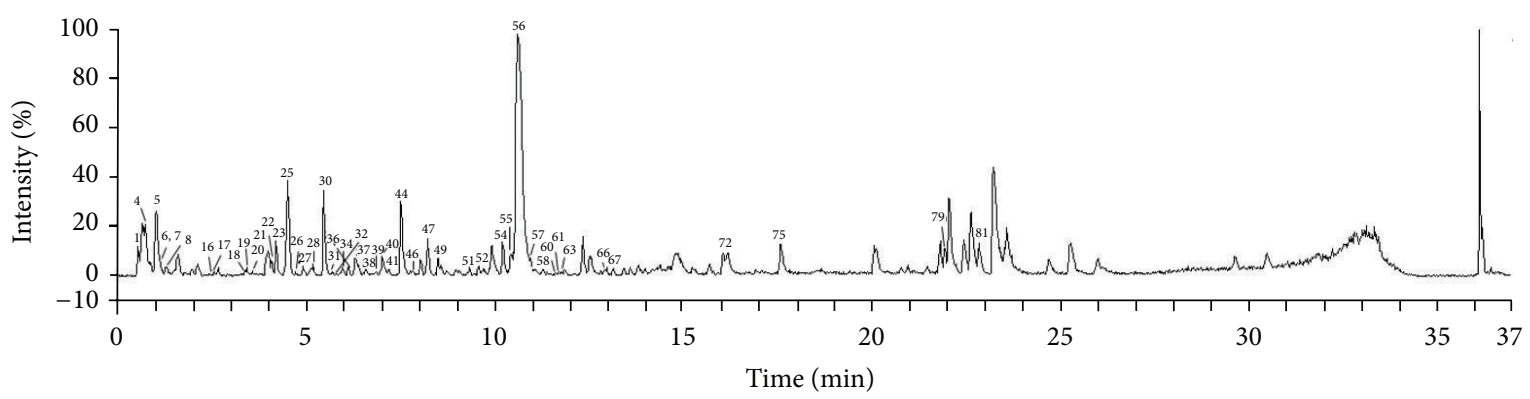

(b)

FIGURE 1: UPLC/Q-TOF-MS analysis of NXT. (a) Chromatograms of NXT in positive ion mode. (b) Chromatograms of NXT in negative ion mode.

used as the collision gas, was maintained at a pressure of $5.3 \times 10^{-5}$ Torr. The instrument was operated with the first resolving quadruple in a wide pass mode (100 Da-1500 Da). Leucineen kephalinamide acetate was used as the lock mass $\left([\mathrm{M}-\mathrm{H}]^{-}=553.2775,[\mathrm{M}+\mathrm{H}]^{+}=555.2931\right)$.

\subsection{Calculation and Prediction of Absorbable Components.} First, we determined the structural formulas of the chemical components that were identified in compound NXT from the Chemical Book website and used the Chemdraw software to draw these formulas. Then, we imported these structural formulas into the Online SMILES Translator and Structure File Generator (http://cactus.nci.nih.gov/translate/) to obtain the smiles format. Finally, we input the smiles format of the chemical components into the Molispiration Smiles website (http://www.molinspiration.com/cgi-bin/properties) to calculate the prediction parameters of drug absorption. According to the five principles of drug absorption, if a component was subject to the following provisions of the corresponding parameters, it could be identified as an absorbable component: hydrogen bond donor (the number of hydrogen atoms attached to the $\mathrm{O}$ and $\mathrm{N}) n \mathrm{OHNH} \leq$ 5; relative molecular mass $\mathrm{MW} \leq 500$; fat water partition coefficient miLog $P \leq 5$; and hydrogen bond acceptor (the number of $\mathrm{O}$ and $\mathrm{N}) n \mathrm{ON} \leq 10$.

2.3. Prediction and Screening of Targets. Using the software of Chembio3D Ultru12.0, we transformed the structure of the absorbed components into the sdf structure format. Then, to predict the possible targets, we imported the components into the public network server of the target database of the efficacy group PharmaMapper website (http://59.78.96.61/pharmmapper/) to perform reverse docking. We selected the top 10 targets for subsequent study.

2.4. Prediction and Screening of Pathways. We imported the obtained targets into the Bio database (http://bioinfo .capitalbio.com $/ \mathrm{mas} 3 /$ ) and then screened for pathways that met the criterion of $P<0.01$.

2.5. Construction of Network. According to the screening pathways with their corresponding targets and components, we created a component-target-pathway illustration using Cytoscape. Then, according to the main selected targets, we drew a target-composition diagram.

\section{Results}

3.1. UPLC/Q-TOF-MS Analysis. We analysed the chemical components of NXT using ultraperformance liquid chromatography combined with quadrupole time-of-flight mass spectrometry. Because different chemical components had better responses in different modes, MS data were obtained in both positive ion mode (Figure 1(a)) and negative ion mode (Figure $1(\mathrm{~b})$ ). MS data in $(+/-)$ ESI modes and the identification results for the constituents in NXT were presented in Table 2. In all 16 herbs from NXT, no related component in Myrrha and Hirudo was found.

3.2. Absorption Parameters of Components. Using a computer prediction method to calculate the identified compounds of NXT, we obtained absorption parameters that could determine whether the chemical compositions could be absorbed. 
TABLE 2: MS data in (+/-) ESI modes and the identification results for the constituents in NXT.

\begin{tabular}{|c|c|c|c|c|c|c|}
\hline Peak number & $\mathrm{RT}(\min )$ & Identification & Mode & $\operatorname{MS}(m / z)$ & Composition & Herbal source \\
\hline 1 & 0.647 & Arginine & Pos/Neg & 174.2024 & $\mathrm{C}_{6} \mathrm{H}_{14} \mathrm{~N}_{4} \mathrm{O}_{2}$ & $\mathrm{PT}$ \\
\hline 2 & 0.702 & Valine & Pos & 117.1478 & $\mathrm{C}_{5} \mathrm{H}_{11} \mathrm{NO}_{2}$ & PT \\
\hline 3 & 0.721 & Proline & Pos & 115.1331 & $\mathrm{C}_{5} \mathrm{H}_{9} \mathrm{NO}_{2}$ & PT \\
\hline 4 & 0.776 & Malic acid & Neg & 134.0911 & $\mathrm{C}_{4} \mathrm{H}_{6} \mathrm{O}_{5}$ & RA \\
\hline 5 & 1.053 & Citric acid & Neg & 192.1286 & $\mathrm{C}_{6} \mathrm{H}_{8} \mathrm{O}_{7}$ & RA \\
\hline 6 & 1.201 & D-5-oxoproline & Neg & 129.1174 & $\mathrm{C}_{5} \mathrm{H}_{7} \mathrm{NO}_{3}$ & RAS \\
\hline 7 & 1.201 & L-5-oxoproline & $\mathrm{Neg}$ & 129.1174 & $\mathrm{C}_{5} \mathrm{H}_{7} \mathrm{NO}_{3}$ & RAS \\
\hline 8 & 1.275 & Succinic acid & Neg & 118.0910 & $\mathrm{C}_{4} \mathrm{H}_{6} \mathrm{O}_{4}$ & RAS, RAB, PT \\
\hline 9 & 1.294 & $\rho$-Coumaric acid & Pos & 164.1601 & $\mathrm{C}_{9} \mathrm{H}_{8} \mathrm{O}_{3}$ & RAS \\
\hline 10 & 1.310 & o-Phthalic acid & Pos & 166.1294 & $\mathrm{C}_{8} \mathrm{H}_{6} \mathrm{O}_{4}$ & RAS \\
\hline 11 & 1.312 & Adenosine & Pos & 267.2403 & $\mathrm{C}_{10} \mathrm{H}_{13} \mathrm{~N}_{5} \mathrm{O}_{4}$ & RAS, PT, RCX \\
\hline 12 & 1.331 & Leucine & Pos & 131.1688 & $\mathrm{C}_{6} \mathrm{H}_{13} \mathrm{NO}_{2}$ & $\mathrm{PT}$ \\
\hline 13 & 1.460 & Isoleucine & Pos & 131.1688 & $\mathrm{C}_{6} \mathrm{H}_{13} \mathrm{NO}_{2}$ & RAB \\
\hline 14 & 1.589 & Gallic acid ${ }^{\mathrm{a}}$ & Neg & 170.1207 & $\mathrm{C}_{7} \mathrm{H}_{6} \mathrm{O}_{5}$ & RPR \\
\hline 15 & 2.199 & Phenylalanine & Pos & 165.1874 & $\mathrm{C}_{9} \mathrm{H}_{11} \mathrm{NO}_{2}$ & $\mathrm{FC}$ \\
\hline 16 & 2.459 & Danshensu & Neg & 198.1701 & $\mathrm{C}_{9} \mathrm{H}_{10} \mathrm{O}_{5}$ & RSM \\
\hline 17 & 2.606 & Palmitic acid & $\mathrm{Neg}$ & 256.3380 & $\mathrm{C}_{16} \mathrm{H}_{32} \mathrm{O}_{2}$ & RAS, FC, RA, SCP \\
\hline 18 & 3.438 & Senkyunolide B & $\mathrm{Neg}$ & 204.2374 & $\mathrm{C}_{12} \mathrm{H}_{12} \mathrm{O}_{3}$ & RCX \\
\hline 19 & 3.456 & Senkyunolide C & Neg & 204.2374 & $\mathrm{C}_{12} \mathrm{H}_{12} \mathrm{O}_{3}$ & RCX \\
\hline 20 & 3.600 & Protocatechuic aldehyde & Neg & 138.1185 & $\mathrm{C}_{7} \mathrm{H}_{6} \mathrm{O}_{3}$ & RSM, RC \\
\hline 21 & 3.974 & Mulberroside $A^{a}$ & Neg & 568.5277 & $\mathrm{C}_{26} \mathrm{H}_{32} \mathrm{O}_{14}$ & $\mathrm{RM}$ \\
\hline 22 & 4.122 & Gallicin & Neg & 184.1453 & $\mathrm{C}_{8} \mathrm{H}_{8} \mathrm{O}_{5}$ & $\mathrm{RPR}$ \\
\hline 23 & 4.230 & Hydroxysafflor yellow A & Pos/Neg & 612.5364 & $\mathrm{C}_{27} \mathrm{H}_{32} \mathrm{O}_{16}$ & FC \\
\hline 24 & 4.232 & 7-Hydroxycoumarin & Pos & 162.1457 & $\mathrm{C}_{9} \mathrm{H}_{6} \mathrm{O}_{3}$ & $\mathrm{RM}$ \\
\hline 25 & 4.565 & Vanillic acid & Neg & 168.1459 & $\mathrm{C}_{8} \mathrm{H}_{8} \mathrm{O}_{4}$ & RCX, RPR \\
\hline 26 & 4.694 & Benzoic acid & Neg & 122.1209 & $\mathrm{C}_{7} \mathrm{H}_{6} \mathrm{O}_{2}$ & RPR \\
\hline 27 & 4.935 & Epicatechin & Neg & 290.2674 & $\mathrm{C}_{15} \mathrm{H}_{14} \mathrm{O}_{6}$ & CS \\
\hline 28 & 5.157 & Catechin & Neg & 290.2674 & $\mathrm{C}_{15} \mathrm{H}_{14} \mathrm{O}_{6}$ & RPR \\
\hline 29 & 5.212 & Albiflorin & Pos & 480.4653 & $\mathrm{C}_{23} \mathrm{H}_{28} \mathrm{O}_{11}$ & $\mathrm{RPR}$ \\
\hline 30 & 5.730 & Quercetin-7-O-glucoside & Neg & 464.3754 & $\mathrm{C}_{21} \mathrm{H}_{20} \mathrm{O}_{12}$ & $\mathrm{FC}$ \\
\hline 31 & 5.952 & Rutin & Neg & 610.5203 & $\mathrm{C}_{27} \mathrm{H}_{30} \mathrm{O}_{16}$ & RA \\
\hline 32 & 5.970 & Calycosin $^{\mathrm{a}}$ & $\mathrm{Neg}$ & 284.2679 & $\mathrm{C}_{16} \mathrm{H}_{12} \mathrm{O}_{5}$ & RA \\
\hline 33 & 5.988 & Calycosin-7-O-glucoside & Pos & 446.4075 & $\mathrm{C}_{22} \mathrm{H}_{22} \mathrm{O}_{10}$ & RA \\
\hline 34 & 5.989 & Ferulic acid ${ }^{\mathrm{a}}$ & Neg & 194.1815 & $\mathrm{C}_{10} \mathrm{H}_{10} \mathrm{O}_{4}$ & RA, RCX, RAS, RAB \\
\hline 35 & 6.321 & Paeoniflorin $^{\mathrm{a}}$ & Pos & 480.466 & $\mathrm{C}_{23} \mathrm{H}_{28} \mathrm{O}_{11}$ & RPR \\
\hline 36 & 6.358 & Pentagalloylglucose $^{\mathrm{a}}$ & Neg & 940.68 & $\mathrm{C}_{41} \mathrm{H}_{32} \mathrm{O}_{26}$ & RPR \\
\hline 37 & 6.413 & Kaempferol-3-O-rutinoside ${ }^{a}$ & Pos/Neg & 594.5179 & $\mathrm{C}_{27} \mathrm{H}_{30} \mathrm{O}_{15}$ & FC \\
\hline 38 & 6.654 & 3,5-Di-O-caffeoylquinic acid & Pos/Neg & 516.4573 & $\mathrm{C}_{25} \mathrm{H}_{24} \mathrm{O}_{12}$ & CS \\
\hline 39 & 6.987 & Dicaffeoylquinic acid & Neg & 516.1275 & $\mathrm{C}_{25} \mathrm{H}_{24} \mathrm{O}_{12}$ & RCX \\
\hline 40 & 7.042 & Z-Butylidenephthalide ${ }^{a}$ & Neg & 188.2259 & $\mathrm{C}_{12} \mathrm{H}_{12} \mathrm{O}_{2}$ & RCX \\
\hline 41 & 7.210 & Salvianolic acid A & Neg & 494.4578 & $\mathrm{C}_{26} \mathrm{H}_{22} \mathrm{O}_{10}$ & RSM \\
\hline 42 & 7.449 & 4-Hydroxyl-3-butylphthalide & Pos & 206.2346 & $\mathrm{C}_{12} \mathrm{H}_{14} \mathrm{O}_{3}$ & RCX \\
\hline 43 & 7.540 & Salvianolic acid B & Neg & 718.6220 & $\mathrm{C}_{36} \mathrm{H}_{30} \mathrm{O}_{16}$ & RSM \\
\hline 44 & 7.688 & Ononin & Pos & 430.4107 & $\mathrm{C}_{22} \mathrm{H}_{22} \mathrm{O}_{9}$ & CS \\
\hline 45 & 7.763 & Senkyunolide F & Pos & 206.1017 & $\mathrm{C}_{12} \mathrm{H}_{14} \mathrm{O}_{3}$ & RCX, RAS \\
\hline 46 & 7.855 & Salvianolic acid E & Neg & 718.1512 & $\mathrm{C}_{36} \mathrm{H}_{30} \mathrm{O}_{16}$ & RSM \\
\hline 47 & 8.243 & Biochanin A & Pos/Neg & 284.2689 & $\mathrm{C}_{16} \mathrm{H}_{12} \mathrm{O}_{5}$ & CS \\
\hline 48 & 8.262 & (6aR,1laR)-3-Hydroxy-9,10-dimethoxy pterocarpan & Pos & 300.3107 & $\mathrm{C}_{17} \mathrm{H}_{16} \mathrm{O}_{5}$ & RA \\
\hline 49 & 8.594 & N1-N5-(Z)-N10-(E)-tri-p-coumaroylspermidine & Pos & 583.2703 & $\mathrm{C}_{34} \mathrm{H}_{37} \mathrm{~N}_{3} \mathrm{O}_{6}$ & $\mathrm{FC}$ \\
\hline
\end{tabular}


TABLE 2: Continued.

\begin{tabular}{|c|c|c|c|c|c|c|}
\hline Peak number & $\mathrm{RT}(\min )$ & Identification & Mode & $\operatorname{MS}(m / z)$ & Composition & Herbal source \\
\hline 50 & 8.740 & Benzoylpaeoniflorin & Pos & 584.5723 & $\mathrm{C}_{30} \mathrm{H}_{32} \mathrm{O}_{12}$ & RPR \\
\hline 51 & 9.518 & Pratensein & $\mathrm{Neg}$ & 300.0679 & $\mathrm{C}_{16} \mathrm{H}_{12} \mathrm{O}_{6}$ & RA \\
\hline 52 & 9.611 & Hydroxyl calendic acid & Neg & 294.4342 & $\mathrm{C}_{18} \mathrm{H}_{30} \mathrm{O}_{3}$ & SP \\
\hline 53 & 9.648 & Trans-oxyresveratrol & Pos & 244.2435 & $\mathrm{C}_{14} \mathrm{H}_{12} \mathrm{O}_{4}$ & $\mathrm{RM}$ \\
\hline 54 & 10.240 & Formononetin $^{\mathrm{a}}$ & Pos/Neg & 268.2580 & $\mathrm{C}_{16} \mathrm{H}_{12} \mathrm{O}_{4}$ & RA \\
\hline 55 & 10.405 & Astragaloside IV & Neg & 784.4633 & $\mathrm{C}_{41} \mathrm{H}_{68} \mathrm{O}_{14}$ & RA \\
\hline 56 & 10.590 & Senkyunolide H & $\mathrm{Neg}$ & 220.2305 & $\mathrm{C}_{12} \mathrm{H}_{12} \mathrm{O}_{4}$ & $\mathrm{RCX}$ \\
\hline 57 & 10.978 & Astragaloside II & Neg & 826.4701 & $\mathrm{C}_{43} \mathrm{H}_{70} \mathrm{O}_{15}$ & RA \\
\hline 58 & 11.311 & Soyasaponin I & Neg & 942.5145 & $\mathrm{C}_{48} \mathrm{H}_{78} \mathrm{O}_{18}$ & RA \\
\hline 59 & 11.422 & Methyl tanshinonate & Pos & 338.1087 & $\mathrm{C}_{20} \mathrm{H}_{18} \mathrm{O}_{5}$ & RSM \\
\hline 60 & 11.588 & Carnosic acid & Neg & 332.4311 & $\mathrm{C}_{20} \mathrm{H}_{28} \mathrm{O}_{4}$ & RSM \\
\hline 61 & 11.644 & Kaempferol-3-O-glucoside & Neg & 448.3752 & $\mathrm{C}_{21} \mathrm{H}_{20} \mathrm{O}_{11}$ & $\mathrm{FC}$ \\
\hline 62 & 11.699 & Hydroxytanshinone IIA & Pos & 310.1199 & $\mathrm{C}_{19} \mathrm{H}_{18} \mathrm{O}_{4}$ & RSM \\
\hline 63 & 11.792 & 3-Butylidene-7-hydroxyphalide & Neg & 204.2331 & $\mathrm{C}_{12} \mathrm{H}_{12} \mathrm{O}_{3}$ & $\mathrm{RCX}$ \\
\hline 64 & 11.921 & Tanshinone II-B & Pos & 310.1187 & $\mathrm{C}_{19} \mathrm{H}_{18} \mathrm{O}_{4}$ & RSM \\
\hline 65 & 12.198 & Senkyunolide A & Pos & 192.2516 & $\mathrm{C}_{12} \mathrm{H}_{16} \mathrm{O}_{2}$ & RCX \\
\hline 66 & 12.975 & Salvianolic acid F & Neg & 314.0735 & $\mathrm{C}_{17} \mathrm{H}_{14} \mathrm{O}_{6}$ & RSM \\
\hline 67 & 13.196 & Kumatakenin & $\mathrm{Neg}$ & 314.3359 & $\mathrm{C}_{17} \mathrm{H}_{14} \mathrm{O}_{6}$ & RA \\
\hline 68 & 13.233 & 3-n-Butylphthalide & Pos & 190.2356 & $\mathrm{C}_{12} \mathrm{H}_{14} \mathrm{O}_{2}$ & RCX \\
\hline 69 & 13.474 & (Z)-ligustilide ${ }^{\mathrm{a}}$ & Pos & 190.2109 & $\mathrm{C}_{12} \mathrm{H}_{14} \mathrm{O}_{2}$ & RAS \\
\hline 70 & 13.483 & (E)-ligustilide ${ }^{a}$ & Pos & 190.2109 & $\mathrm{C}_{12} \mathrm{H}_{14} \mathrm{O}_{2}$ & RAS \\
\hline 71 & 13.917 & Trijuganone B & Pos & 280.1107 & $\mathrm{C}_{18} \mathrm{H}_{16} \mathrm{O}_{3}$ & RSM \\
\hline 72 & 16.098 & Cryptotanshinone $^{\mathrm{a}}$ & Neg & 296.3642 & $\mathrm{C}_{19} \mathrm{H}_{20} \mathrm{O}_{3}$ & RSM \\
\hline 73 & 16.394 & Senkyunolide M & Pos & 278.1565 & $\mathrm{C}_{16} \mathrm{H}_{22} \mathrm{O}_{4}$ & $\mathrm{RCX}$ \\
\hline 74 & 17.503 & O-Phthalic anhydride & Pos & 148.0207 & $\mathrm{C}_{8} \mathrm{H}_{4} \mathrm{O}_{3}$ & $\mathrm{FC}$ \\
\hline 75 & 17.614 & Chlorogenic acid ${ }^{\mathrm{a}}$ & Neg & 354.3120 & $\mathrm{C}_{16} \mathrm{H}_{18} \mathrm{O}_{9}$ & CS \\
\hline 76 & 18.076 & Tanshinone IIA & Pos & 294.3430 & $\mathrm{C}_{19} \mathrm{H}_{18} \mathrm{O}_{3}$ & RSM \\
\hline 77 & 18.205 & Angelicide & Pos & 380.1917 & $\mathrm{C}_{24} \mathrm{H}_{28} \mathrm{O}_{4}$ & RCX \\
\hline 78 & 20.460 & Carthamidin & Pos & 288.2575 & $\mathrm{C}_{15} \mathrm{H}_{12} \mathrm{O}_{6}$ & $\mathrm{FC}$ \\
\hline 79 & 22.078 & Linoleic acid & $\mathrm{Neg}$ & 280.2387 & $\mathrm{C}_{18} \mathrm{H}_{32} \mathrm{O}_{2}$ & SP \\
\hline 80 & 22.659 & Acetyl-11-keto- $\beta$-boswellic acid & Pos/Neg & 512.7458 & $\mathrm{C}_{32} \mathrm{H}_{48} \mathrm{O}_{5}$ & FK \\
\hline 81 & 22.881 & Oleanolic acid & Neg & 456.3652 & $\mathrm{C}_{30} \mathrm{H}_{48} \mathrm{O}_{3}$ & RSM \\
\hline
\end{tabular}

"a" refers to the component has been verified by standard substance.

Table 3 showed the specific absorption parameters of all of the components. The data indicated that there were a total of 63 chemical compositions (Figure 2) that met the five principles of drug absorption. As shown, 7 glycosides were identified. Although the relative molecular masses of those compounds were greater than 500, they could also be absorbed, because those compounds could be divided into two parts, including aglycones which mainly mediated drug efficacy and sugar chains in the body. So we could import these glycosides' aglycones into PharmMapper to obtain the relevant parameters. The results showed that both of these components were consistent with the five principles of drug absorption, so we considered that these 7 chemical compositions could be absorbed in the body.

3.3. Potential Targets and Pathways. By importing 63 chemical compositions that were predicted to be absorbable into the PharmMapper database for directional docking, we obtained a total of 123 targets. We then imported these targets into the Molecule Annotation System and obtained 77 pathways regulated by NXT with highly significant differences, from which we chose the top 40 pathways that met the criterion of $P<0.01$ (Table 4 ). A total of 34 targets were related to these top 40 pathways, and HRAS, MAP2K1, and MAPK14 were associated with most of these pathways, so we considered these factors to be the main targets. As shown in Table 4, NFAT and hypertrophy of the heart (transcription in the broken heart) ranked first among these pathways.

In Table 5, these top 40 pathways were classified into 5 categories, which included pathways associated with heart diseases and blood vessels, metabolism, cell cycle (with proliferation and apoptosis), immunity, and other pathways. By classifying these pathways, we accessed and marked the corresponding medicinal materials of NXT (Table 5). In the pathways associated with heart diseases and blood vessels, 
TABLE 3: Absorption parameters of the components.

\begin{tabular}{|c|c|c|c|c|c|c|}
\hline Number & Compounds & MW & $n \mathrm{ON}$ & $n \mathrm{OHNH}$ & $\operatorname{miLog} P$ & Results \\
\hline 1 & Arginine & 174.204 & 6 & 7 & -3.632 & $x$ \\
\hline 2 & Valine & 117.15 & 3 & 3 & -1.91 & $\sqrt{ }$ \\
\hline 3 & Proline & 115.132 & 3 & 2 & -1.723 & $\sqrt{ }$ \\
\hline 4 & Malic acid & 134.087 & 5 & 3 & -1.57 & $\sqrt{ }$ \\
\hline 5 & Citric acid & 192.123 & 7 & 4 & -1.983 & $\sqrt{ }$ \\
\hline 6 & D-5-oxoproline & 129.115 & 4 & 2 & -2.402 & $\sqrt{ }$ \\
\hline 7 & L-5-oxoproline & 129.115 & 4 & 2 & -2.402 & $\sqrt{ }$ \\
\hline 8 & Succinic acid & 118.088 & 4 & 2 & -0.655 & $\sqrt{ }$ \\
\hline 9 & $\rho$-Coumaric acid & 164.160 & 3 & 2 & 1.43 & $\sqrt{ }$ \\
\hline 10 & o-Phthalic acid & 166.132 & 4 & 2 & 1.034 & $\sqrt{ }$ \\
\hline 11 & Adenosine & 267.245 & 9 & 5 & -0.854 & $\sqrt{ }$ \\
\hline 12 & Leucine & 131.175 & 3 & 3 & -1.382 & $\sqrt{ }$ \\
\hline 13 & Isoleucine & 131.175 & 3 & 3 & -1.41 & $\sqrt{ }$ \\
\hline 14 & Gallic acid ${ }^{\mathrm{a}}$ & 170.120 & 5 & 4 & 0.589 & $\sqrt{ }$ \\
\hline 15 & Phenylalanine & 165.192 & 3 & 3 & -1.231 & $\sqrt{ }$ \\
\hline 16 & Danshensu & 198.174 & 5 & 4 & -0.251 & $\sqrt{ }$ \\
\hline 17 & Palmitic acid & 256.43 & 2 & 1 & 7.059 & $\times$ \\
\hline 18 & Senkyunolide B & 204.225 & 3 & 1 & 2.81 & $\sqrt{ }$ \\
\hline 19 & Senkyunolide C & 204.225 & 3 & 1 & 2.574 & $\sqrt{ }$ \\
\hline 20 & Protocatechuic aldehyde & 138.122 & 3 & 2 & 0.759 & $\sqrt{ }$ \\
\hline 21 & Mulberroside $\mathrm{A}^{\mathrm{a}}$ & 568.528 & 14 & 10 & -0.852 & $\sqrt{ }$ \\
\hline 22 & Gallicin & 184.147 & 5 & 3 & 0.848 & $\sqrt{ }$ \\
\hline 23 & Hydroxysafflor yellow A & 612.54 & 16 & 12 & -4.12 & $\sqrt{ }$ \\
\hline 24 & 7-Hydroxycoumarin & 162.144 & 3 & 1 & 1.511 & $\sqrt{ }$ \\
\hline 25 & Vanillic acid & 168.148 & 4 & 2 & 1.187 & $\sqrt{ }$ \\
\hline 26 & Benzoic acid & 122.123 & 2 & 1 & 1.848 & $\sqrt{ }$ \\
\hline 27 & Epicatechin & 290.271 & 6 & 5 & 1.369 & $\sqrt{ }$ \\
\hline 28 & Catechin & 290.271 & 6 & 5 & 1.369 & $\sqrt{ }$ \\
\hline 29 & Albiflorin & 480.466 & 11 & 5 & -1.636 & $\times$ \\
\hline 30 & Quercetin-7-O-glucoside & 464.379 & 12 & 8 & -0.104 & $\times$ \\
\hline 31 & Rutin & 610.521 & 16 & 10 & -1.063 & $\sqrt{ }$ \\
\hline 32 & Calycosin $^{a}$ & 284.267 & 5 & 2 & 2.377 & $\sqrt{ }$ \\
\hline 33 & Calycosin-7-O-glucoside & 446.408 & 10 & 5 & 0.59 & $\sqrt{ }$ \\
\hline 34 & Ferulic acid ${ }^{\mathrm{a}}$ & 194.186 & 4 & 2 & 1.249 & $\sqrt{ }$ \\
\hline 35 & Paeoniflorin ${ }^{\mathrm{a}}$ & 480.466 & 11 & 5 & 0.044 & $\times$ \\
\hline 36 & Pentagalloylglucose $\mathrm{e}^{\mathrm{a}}$ & 940.681 & 26 & 15 & 2.761 & $\sqrt{ }$ \\
\hline 37 & Kaempferol-3-O-rutinoside ${ }^{a}$ & 594.522 & 15 & 9 & -0.574 & $\sqrt{ }$ \\
\hline 38 & 3,5-Di-O-caffeoylquinic acid ${ }^{\mathrm{a}}$ & 516.455 & 12 & 7 & 1.424 & $x$ \\
\hline 39 & Dicaffeoylquinic acid & 516.46 & 12 & 7 & 1.21 & $\times$ \\
\hline 40 & Z-Butylidenephthalide ${ }^{a}$ & 188.226 & 2 & 0 & 3.077 & $\sqrt{ }$ \\
\hline 41 & Salvianolic acid A & 494.452 & 10 & 7 & 3.014 & $\times$ \\
\hline 42 & 4-Hydroxyl-3-butylphthalide & 206.241 & 3 & 1 & 3.42 & $\sqrt{ }$ \\
\hline 43 & Salvianolic acid B & 718.620 & 16 & 9 & 1.615 & $\times$ \\
\hline 44 & Ononin & 430.409 & 9 & 4 & 1.307 & $\sqrt{ }$ \\
\hline 45 & Senkyunolide F & 206.24 & 3 & 1 & 1.72 & $\sqrt{ }$ \\
\hline 46 & Salvianolic acid E & 718.62 & 16 & 10 & 2.83 & $\times$ \\
\hline 47 & Biochanin A & 284.267 & 5 & 2 & 2.804 & $\sqrt{ }$ \\
\hline 48 & $(6 \mathrm{aR}, 11 \mathrm{aR})-3-$ Hydroxy-9,10-dimethoxy pterocarpan & 300.31 & 5 & 1 & 2.546 & $\sqrt{ }$ \\
\hline 49 & N1-N5-(Z)-N10-(E)-tri-p-coumaroylspermidine & 538.68 & 9 & 5 & 4.3 & $\times$ \\
\hline 50 & Benzoylpaeoniflorin & 584.574 & 12 & 4 & 2.472 & $\times$ \\
\hline 51 & Pratensein & 300.27 & 6 & 3 & 2.09 & $\sqrt{ }$ \\
\hline
\end{tabular}


TABLE 3: Continued.

\begin{tabular}{|c|c|c|c|c|c|c|}
\hline Number & Compounds & MW & $n \mathrm{ON}$ & $n \mathrm{OHNH}$ & $\operatorname{miLog} P$ & Results \\
\hline 52 & Hydroxyl calendic acid & 294.435 & 3 & 2 & 4.93 & $\sqrt{ }$ \\
\hline 53 & Trans-Oxyresveratrol & 244.246 & 4 & 4 & 2.723 & $\sqrt{ }$ \\
\hline 54 & Formononetin $^{\mathrm{a}}$ & 268.268 & 4 & 1 & 3.095 & $\sqrt{ }$ \\
\hline 55 & Astragaloside IV & 784.98 & 14 & 9 & 1.21 & $\sqrt{ }$ \\
\hline 56 & Senkyunolide H & 220.224 & 4 & 2 & 2.314 & $\sqrt{ }$ \\
\hline 57 & Astragaloside II & 827.02 & 15 & 8 & 1.91 & $\sqrt{ }$ \\
\hline 58 & Soyasaponin I & 943.13 & 18 & 11 & 1.7 & $x$ \\
\hline 59 & Methyl tanshinonate & 338.36 & 5 & 0 & 0.93 & $\sqrt{ }$ \\
\hline 60 & Carnosic acid & 332.440 & 4 & 3 & 4.603 & $\sqrt{ }$ \\
\hline 61 & Kaempferol-3-O-glucoside & 448.380 & 11 & 7 & 0.125 & $x$ \\
\hline 62 & Hydroxytanshinone IIA & 310.35 & 4 & 1 & 3.24 & $\sqrt{ }$ \\
\hline 63 & 3-Butylidene-7-hydroxyphthalide & 204.225 & 3 & 1 & 2.81 & $\sqrt{ }$ \\
\hline 64 & Tanshinone II-B & 310.35 & 4 & 1 & 2.97 & $\sqrt{ }$ \\
\hline 65 & Senkyunolide A & 192.258 & 2 & 0 & 3.521 & $\sqrt{ }$ \\
\hline 66 & Salvianolic acid F & 314.29 & 6 & 5 & 2.33 & $\sqrt{ }$ \\
\hline 67 & Kumatakenin & 314.29 & 6 & 2 & 2.98 & $\sqrt{ }$ \\
\hline 68 & 3-n-Butylphthalide & 190.242 & 2 & 0 & 3.483 & $\sqrt{ }$ \\
\hline 69 & (Z)-Ligustilide ${ }^{\mathrm{a}}$ & 190.242 & 2 & 0 & 2.927 & $\sqrt{ }$ \\
\hline 70 & (E)-Ligustilide ${ }^{\mathrm{a}}$ & 190.242 & 2 & 0 & 2.927 & $\sqrt{ }$ \\
\hline 71 & Trijuganone B & 280.32 & 3 & 1 & 3.9 & $\sqrt{ }$ \\
\hline 72 & Cryptotanshinone $^{a}$ & 296.366 & 3 & 0 & 3.83 & $\sqrt{ }$ \\
\hline 73 & Senkyunolide M & 278.35 & 4 & 1 & 2.55 & $\sqrt{ }$ \\
\hline 74 & O-Phthalic anhydride & 148.12 & 3 & 0 & 0.93 & $\sqrt{ }$ \\
\hline 75 & Chlorogenic acid ${ }^{\mathrm{a}}$ & 354.311 & 9 & 6 & -0.453 & $x$ \\
\hline 76 & Tanshinone IIA & 294.350 & 3 & 0 & 4.158 & $\sqrt{ }$ \\
\hline 77 & Angelicide & 380.48 & 4 & 0 & 5.73 & $\times$ \\
\hline 78 & Carthamidin & 288.255 & 6 & 4 & 1.649 & $\sqrt{ }$ \\
\hline 79 & Linoleic acid & 280.45 & 2 & 1 & 6.86 & $x$ \\
\hline 80 & Acetyl-11-keto- $\beta$-boswellic acid & 512.73 & 5 & 1 & 6.39 & $\times$ \\
\hline 81 & Oleanolic acid & 456.71 & 3 & 2 & 6.72 & $\times$ \\
\hline
\end{tabular}

Note. " $\sqrt{ }$ " means that component could be absorbed; " $x$ " means that component could not be absorbed.

"a" refers to the component has been verified by standard substance.

RCX, RSM, and FC were the most important. In the regulation of metabolism, RA, RSM, and RCX showed diametrical effect. All the herbs except Semen Persicae (SP) were related metabolism pathways due to the current research. RA, RSM, RCX, and FC could regulate the pathways about cell cycle, proliferation, and apoptosis. Some other important pathways were also affected by some herbs like RA, RSM, and RCX, for example, Insulin Signaling Pathway and p38 MAPK Signaling Pathway.

3.4. Pharmacology Network of NXT. Using the Cytoscape software, we constructed a pharmacology network of NXT (Figure 3), which showed us the relationships of the top 40 pathways, targets, and chemical components. We obtained preliminary understanding of the mechanism of NXT through this network.

In this research, we found three major targets of NXT: HRAS, MAP2K1, and MAPK14, which were involved in most regulated pathways. By Figure 4, based on illustration of the main targets with their corresponding compounds, we found the most effective ingredients of NXT were organic acids, saponins, and tanshinones. The main sources of organic acids were RA, RCX, RAS, and RAB. The saponins were mainly derived from RA. Meanwhile, tanshinones were mainly concentrated in RSM.

\section{Discussion}

The burden of cardiovascular and circulatory disease is becoming more and more serious, with cerebrovascular disease $(\mathrm{CBD})$ and ischemic heart disease being the most serious [7]. As the causes of cardiovascular disease (CVD) and $\mathrm{CBD}$ are complicated, the symptoms of these diseases are also very diverse. NXT is commonly used during clinical treatment of CVD and CBD, and the effect of this drug is remarkable. Although complex traditional Chinese medicine has great significance for the treatment of complex diseases, some questions such as the material basis and the potential mechanisms remain unanswered. 
TABLE 4: Top 40 Biocarta pathways regulated by NXT $(P<0.01)$.

\begin{tabular}{|c|c|c|c|c|c|}
\hline Rank & Pathway & Count & $P$-value & $q$-value & Gene \\
\hline 1 & $\begin{array}{l}\text { NFAT and hypertrophy of the heart (transcription in } \\
\text { the broken heart) }\end{array}$ & 6 & $5.75 E-10$ & $3.58 E-09$ & $\begin{array}{l}\text { HRAS; GSK3B; MAPK14; } \\
\text { FKBP1A; F2; MAP2K1 }\end{array}$ \\
\hline 2 & Phosphoinositides and their downstream targets & 5 & $1.39 E-09$ & $8.47 E-09$ & $\begin{array}{l}\text { GSK3B; PDPK1; BTK; RAB5A; } \\
\text { EEA1 }\end{array}$ \\
\hline 3 & Intrinsic Prothrombin Activation Pathway & 4 & $8.50 E-08$ & $2.82 E-07$ & F10; FGG; F11; F2 \\
\hline 4 & Bioactive Peptide Induced Signaling Pathway & 4 & $4.08 E-07$ & $9.30 E-07$ & HRAS; MAPK14; F2; MAP2K1 \\
\hline 5 & BCR Signaling Pathway & 4 & $4.88 E-07$ & $1.08 E-06$ & $\begin{array}{l}\text { HRAS; MAPK14; BTK; } \\
\text { MAP2K1 }\end{array}$ \\
\hline 6 & $\begin{array}{l}\text { Estrogen-responsive protein Efp controls cell cycle and } \\
\text { breast tumors growth }\end{array}$ & 3 & $6.40 E-07$ & $1.34 E-06$ & CDK2; ESR1; CDK6 \\
\hline 7 & Nuclear receptors in lipid metabolism and toxicity & 4 & $8.02 E-07$ & $1.58 E-06$ & $\begin{array}{l}\text { CYP2C9; VDR; NR1H3; } \\
\text { PPARA }\end{array}$ \\
\hline 8 & Map kinase inactivation of SMRT corepressor & 3 & $1.53 E-06$ & $2.48 E-06$ & THRB; MAPK14; MAP2K1 \\
\hline 9 & MAP Kinase Signaling Pathway & 5 & $2.09 E-06$ & $3.05 E-06$ & $\begin{array}{l}\text { HRAS; MAPK10; MAPK14; } \\
\text { TGFBR1; MAP2K1 }\end{array}$ \\
\hline 10 & Extrinsic Prothrombin Activation Pathway & 3 & $2.99 E-06$ & $4.05 E-06$ & F10; FGG; F2 \\
\hline 11 & amiPathway & 3 & $5.17 E-06$ & $6.40 E-06$ & F10; FGG; F2 \\
\hline 12 & $\begin{array}{l}\text { Roles of } \beta \text {-arrestin-dependent recruitment of Src } \\
\text { kinases in GPCR signaling }\end{array}$ & 3 & $6.57 E-06$ & $7.86 E-06$ & HRAS; HCK; MAP2K1 \\
\hline 13 & $\begin{array}{l}\text { Aspirin blocks signaling pathway involved in platelet } \\
\text { activation }\end{array}$ & 3 & $8.19 E-06$ & $9.49 E-06$ & HRAS; F2; MAP2K1 \\
\hline 14 & Insulin Signaling Pathway & 3 & $2.03 E-05$ & $2.03 E-05$ & HRAS; INSR; MAP2K1 \\
\hline 15 & IL-2 Signaling Pathway & 3 & $2.37 E-05$ & $2.29 E-05$ & HRAS; MAP2K1; LCK \\
\hline 16 & Role of ERBB2 in signal transduction and oncology & 3 & $2.37 E-05$ & $2.29 E-05$ & HRAS; ESR1; MAP2K1 \\
\hline 17 & Links between Pyk2 and MAP kinases & 3 & $2.74 E-05$ & $2.45 E-05$ & HRAS; MAPK14; MAP2K1 \\
\hline 18 & $\begin{array}{l}\text { NF- } \kappa \text { B activation by nontypeable Hemophilus } \\
\text { influenzae }\end{array}$ & 3 & $2.74 E-05$ & $2.45 E-05$ & MAPK14; TGFBR1; NR3C1 \\
\hline 19 & Influence of Ras and Rho proteins on G1 to S transition & 3 & $3.14 E-05$ & $2.82 E-05$ & HRAS; CDK2; CDK6 \\
\hline 20 & $\begin{array}{l}\text { fMLP induced chemokine gene expression in HMC-1 } \\
\text { cells }\end{array}$ & 3 & $3.14 E-05$ & $2.82 E-05$ & HRAS; MAPK14; MAP2K1 \\
\hline 21 & Growth Hormone Signaling Pathway & 3 & $3.14 E-05$ & $2.82 E-05$ & HRAS; INSR; MAP2K1 \\
\hline 22 & Cell cycle: G1/S checkpoint & 3 & $4.06 E-05$ & $3.37 E-05$ & CDK2; GSK3B; CDK6 \\
\hline 23 & Fc epsilon receptor I signaling in mast cells & 3 & $4.58 E-05$ & $3.70 E-05$ & HRAS; BTK; MAP2K1 \\
\hline 24 & Signaling of hepatocyte growth factor receptor & 3 & $6.40 E-05$ & $4.89 E-05$ & HRAS; MET; MAP2K1 \\
\hline 25 & p38 MAPK signaling pathway & 3 & $7.85 E-05$ & $5.76 E-05$ & HRAS; MAPK14; TGFBR1 \\
\hline 26 & Keratinocyte differentiation & 3 & $1.13 E-04$ & $7.81 E-05$ & HRAS; MAPK14; MAP2K1 \\
\hline 27 & $\mathrm{~T}$ cell receptor signaling pathway & 3 & $1.13 E-04$ & $7.81 E-05$ & HRAS; MAP2K1; LCK \\
\hline 28 & $\begin{array}{l}\text { TSP-1 induced apoptosis in microvascular endothelial } \\
\text { cell }\end{array}$ & 2 & $1.46 E-04$ & $9.59 E-05$ & CASP3; MAPK14 \\
\hline 29 & The role of FYVE-finger proteins in vesicle transport & 2 & $1.46 E-04$ & $9.59 E-05$ & RAB5A; EEA1 \\
\hline 30 & $\begin{array}{l}\text { Mechanism of gene regulation by peroxisome } \\
\text { proliferators via PPARa(alpha) }\end{array}$ & 3 & $1.82 E-04$ & $1.15 E-04$ & HSP90AA1; NR1H3; PPARA \\
\hline 31 & Visceral fat deposits and the metabolic syndrome & 2 & $1.95 E-04$ & $1.21 E-04$ & HSD11B1; NR3C1 \\
\hline 32 & $\begin{array}{l}\text { RB tumor suppressor/checkpoint signaling in response } \\
\text { to DNA damage }\end{array}$ & 2 & $2.50 E-04$ & $1.44 E-04$ & CDK2; CHEK1 \\
\hline 33 & Platelet Amyloid Precursor Protein Pathway & 2 & $2.50 E-04$ & $1.44 E-04$ & F11; F2 \\
\hline 34 & Fibrinolysis Pathway & 2 & $3.12 E-04$ & $1.77 E-04$ & FGG; F2 \\
\hline 35 & Corticosteroids and cardioprotection & 2 & $3.12 E-04$ & $1.77 E-04$ & HSP90AA1; NR3C1 \\
\hline 36 & $\begin{array}{l}\text { Phosphorylation of MEK } 1 \text { by } c \mathrm{dk} 5 / \mathrm{p} 35 \text { downregulates } \\
\text { the MAP kinase pathway }\end{array}$ & 2 & $3.81 E-04$ & $2.09 E-04$ & HRAS; MAP2K1 \\
\hline
\end{tabular}


TABLE 4: Continued.

\begin{tabular}{llccll}
\hline Rank & Pathway & Count & $P$-value & $q$-value & Gene \\
\hline 37 & VEGF, hypoxia, and angiogenesis & 2 & $5.38 E-04$ & $2.79 E-04$ & HRAS; KDR \\
38 & How progesterone initiates oocyte membrane & 2 & $6.27 E-04$ & $3.17 E-04$ & HRAS; PGR \\
39 & IL-3 Signaling Pathway & 2 & $6.27 E-04$ & $3.17 E-04$ & HRAS; MAP2K1 \\
40 & Sprouty regulation of tyrosine kinase signals & 2 & $6.27 E-04$ & $3.17 E-04$ & HRAS; MAP2K1 \\
\hline
\end{tabular}

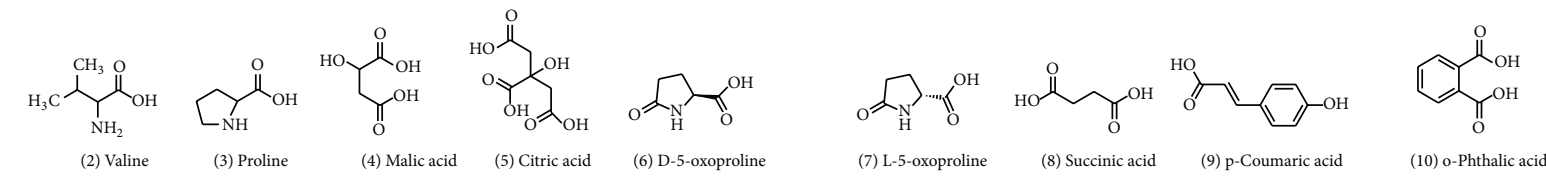<smiles>Nc1ncnn1C1OC(CO)C(O)C1O</smiles><smiles>[2H]C(C)C(N)C(N)C(=O)O</smiles>

(11) Adenosine

(12) Leucine, $\mathrm{R}_{1}=\mathrm{CH}_{3}, \mathrm{R}_{2}=\mathrm{H}$ (13) Isoleucine, $\mathrm{R}_{1}=\mathrm{H}, \mathrm{R}_{2}=\mathrm{CH}$
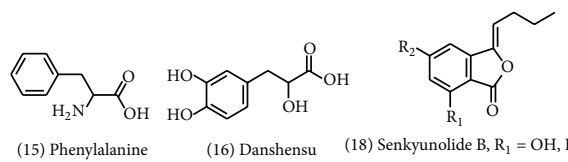

$$
\begin{aligned}
& \text { (18) Senkyunolide B, } R_{1}=O H, R_{2}=H \\
& \text { (19) Senkyunolide C, } R_{1}=H, R_{2}=O H \\
& \text { (40) Z-Butylidenephthalide, } R_{1}=H, R_{2}=H
\end{aligned}
$$<smiles>O=C(CCc1ccc(O)cc1)C1=C(O)C(=C(O)C2OC(CO)C(O)C(O)C2O)C(O)C2OC(CO)C(O)C(O)C2O1</smiles>

(23) Hydroxysafflor yellow A

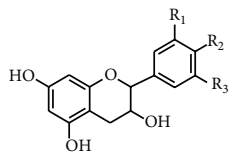

(27) Epicatechin, $\mathrm{R}_{1}=\mathrm{H}, \mathrm{R}_{2}=\mathrm{OH}, \mathrm{R}_{3}=\mathrm{OH}$ (28) Catechin, $\mathrm{R}_{1}=\mathrm{OH}, \mathrm{R}_{2}=\mathrm{OH}, \mathrm{R}_{3}=\mathrm{H}$

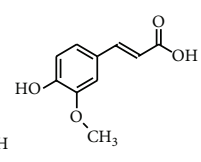<smiles>O=C(OCC1OC(OC(=O)c2cc(O)c(O)c(O)c2)C(OC(=O)c2cc(O)c(O)c(O)c2)C(OC(=O)c2cc(O)c(O)c(O)c2)C1OC(=O)c1cc(O)c(O)c(O)c1)c1cc(O)c(O)c(O)c1</smiles>

(36) Pentagalloylglucosea

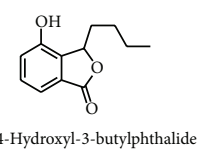

42) 4-Hydroxyl-3-butylphthalide

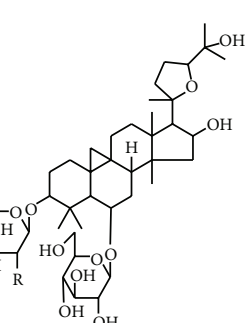

(55) Astragaloside IV, $\mathrm{R}=\mathrm{OH}$ (57) Astragaloside II, $\mathrm{R}=\mathrm{CH}_{3} \mathrm{COO}^{-}$

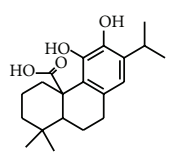

(60) Carnosic acid

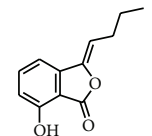
(63) 3-Butylidene-7-
hydroxyphthalide

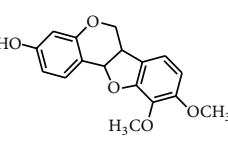

(48) (6aR,11aR)-3-hydroxy-9, 10-dimethoxy pterocarpan

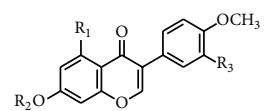

(32) Calycosin ${ }^{\mathrm{a}}, \mathrm{R}_{1}=\mathrm{H}, \mathrm{R}_{2}=\mathrm{H}, \mathrm{R}_{3}=\mathrm{OH}$ (33) Calycosin-7-O-glucoside, $\mathrm{R}_{1}=\mathrm{H}$,

$$
\mathrm{R}_{2}=\mathrm{Glc}, \mathrm{R}_{3}=\mathrm{OH}
$$

(44) Ononin $R_{1}=H_{1} R_{2}=G l c_{3}=H$ (47) Biochanin $\mathrm{A}, \mathrm{R}_{1}=\mathrm{OH}, \mathrm{R}_{2}=\mathrm{H}, \mathrm{R}_{3}=\mathrm{H}$ (51) Pratensein, $\mathrm{R}_{1}=\mathrm{OH}, \mathrm{R}_{2}=\mathrm{H}, \mathrm{R}_{3}=\mathrm{OH}$ (54) Formononetin ${ }^{\mathrm{a}}, \mathrm{R}_{1}=\mathrm{H}, \mathrm{R}_{2}=\mathrm{H}, \mathrm{R}_{3}=\mathrm{H}$<smiles>[R]C(=O)c1cc(Br)c(N)c(Br)c1</smiles>

(14) Gallic acid ${ }^{2}, \mathrm{R}_{1}=\mathrm{OH}, \mathrm{R}_{2}=\mathrm{OH}$

$$
\mathrm{R}_{3}=\mathrm{OH}, \mathrm{R}_{4}=\mathrm{OH}
$$

20) Protocatechuic aldehyde, $\mathrm{R}_{1}=\mathrm{OH}$

$\mathrm{R}_{2}=\mathrm{OH}, \mathrm{R}_{3}=\mathrm{H}, \mathrm{R}_{4}=\mathrm{H}$

(22) Gallicin, $\mathrm{R}_{1}=\mathrm{OH}, \mathrm{R}_{2}=\mathrm{OH}$,

$\mathrm{R}_{3}=\mathrm{OH}, \mathrm{R}_{4}=\mathrm{OCH}_{3}$

(25) Vanillic acid, $\mathrm{R}_{1}=\mathrm{OCH}_{3}, \mathrm{R}_{2}=\mathrm{OH}$

$\mathrm{R}_{3}=\mathrm{H}, \mathrm{R}_{4}=\mathrm{OH}$

(26) Benzoic acid, $\mathrm{R}_{1}=\mathrm{H}, \mathrm{R}_{2}=\mathrm{H}$

$\mathrm{R}_{3}=\mathrm{H}, \mathrm{R}_{4}=\mathrm{COOH}$

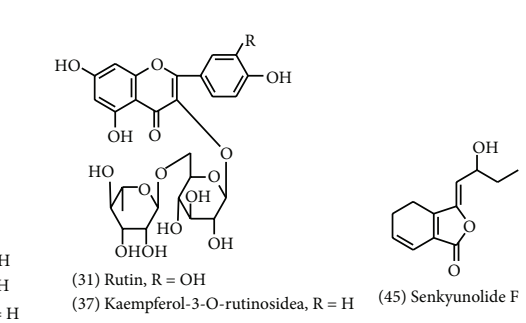


TABLE 5: The herbs of NXT involved in the top 40 pathways.

\begin{tabular}{|c|c|c|c|c|c|c|c|c|c|c|c|c|c|c|}
\hline Category & Pathway & NXT & RA & RPR & RSM & RAS & RCX & SP & FC & CS & RAB & $\mathrm{RC}$ & $\mathrm{RM}$ & PT \\
\hline \multirow{9}{*}{$\begin{array}{l}\text { Pathway associated } \\
\text { with heart diseases } \\
\text { and blood vessels }\end{array}$} & $\begin{array}{l}\text { NFAT and hypertrophy of } \\
\text { the heart (transcription in } \\
\text { the broken heart) }\end{array}$ & 1 & 1 & 1 & 1 & 1 & 1 & 0 & 1 & 1 & 1 & 1 & 1 & 1 \\
\hline & $\begin{array}{l}\text { Intrinsic Prothrombin } \\
\text { Activation Pathway }\end{array}$ & 1 & 0 & 1 & 1 & 1 & 1 & 0 & 1 & 0 & 0 & 0 & 1 & 0 \\
\hline & $\begin{array}{l}\text { Extrinsic Prothrombin } \\
\text { Activation Pathway }\end{array}$ & 1 & 0 & 1 & 1 & 1 & 1 & 0 & 1 & 0 & 0 & 0 & 0 & 0 \\
\hline & $\begin{array}{l}\text { Aspirin blocks signaling } \\
\text { pathway involved in platelet } \\
\text { activation }\end{array}$ & 1 & 1 & 1 & 1 & 1 & 1 & 0 & 1 & 1 & 1 & 1 & 1 & 1 \\
\hline & $\begin{array}{l}\text { TSP-1 induced apoptosis in } \\
\text { microvascular endothelial } \\
\text { cell }\end{array}$ & 1 & 1 & 1 & 1 & 1 & 1 & 0 & 1 & 0 & 0 & 0 & 0 & 0 \\
\hline & $\begin{array}{c}\text { Platelet Amyloid Precursor } \\
\text { Protein Pathway }\end{array}$ & 1 & 0 & 0 & 0 & 0 & 1 & 0 & 1 & 0 & 0 & 0 & 1 & 0 \\
\hline & Fibrinolysis Pathway & 1 & 0 & 1 & 1 & 0 & 1 & 0 & 1 & 0 & 0 & 1 & 0 & 0 \\
\hline & $\begin{array}{l}\text { Corticosteroids and } \\
\text { cardioprotection }\end{array}$ & 1 & 1 & 0 & 1 & 0 & 1 & 0 & 0 & 0 & 0 & 0 & 1 & 0 \\
\hline & $\begin{array}{l}\text { VEGF, hypoxia, and } \\
\text { angiogenesis }\end{array}$ & 1 & 1 & 1 & 1 & 1 & 1 & 1 & 1 & 1 & 1 & 1 & 1 & 1 \\
\hline \multirow{3}{*}{$\begin{array}{l}\text { Pathway associated } \\
\text { with metabolism }\end{array}$} & $\begin{array}{l}\text { Nuclear receptors in lipid } \\
\text { metabolism and toxicity }\end{array}$ & 1 & 1 & 1 & 1 & 0 & 1 & 1 & 1 & 0 & 1 & 0 & 1 & 0 \\
\hline & $\begin{array}{l}\text { Growth Hormone } \\
\text { Signaling Pathway }\end{array}$ & 1 & 1 & 1 & 1 & 1 & 1 & 0 & 1 & 1 & 1 & 1 & 1 & 1 \\
\hline & $\begin{array}{l}\text { Visceral fat deposits and } \\
\text { the metabolic syndrome }\end{array}$ & 1 & 1 & 0 & 1 & 1 & 1 & 0 & 0 & 0 & 0 & 0 & 0 & 0 \\
\hline \multirow{4}{*}{$\begin{array}{l}\text { Pathway associated } \\
\text { with immunity }\end{array}$} & BCR Signaling Pathway & 1 & 1 & 1 & 1 & 1 & 1 & 0 & 1 & 1 & 1 & 1 & 1 & 1 \\
\hline & IL-2 Signaling Pathway & 1 & 1 & 1 & 1 & 1 & 1 & 0 & 1 & 1 & 1 & 1 & 1 & 1 \\
\hline & $\begin{array}{l}\text { fMLP induced chemokine } \\
\text { gene expression in HMC-1 } \\
\text { cells }\end{array}$ & 1 & 1 & 1 & 1 & 1 & 1 & 0 & 1 & 1 & 1 & 1 & 1 & 1 \\
\hline & $\begin{array}{c}\text { T Cell Receptor Signaling } \\
\text { Pathway }\end{array}$ & 1 & 1 & 1 & 1 & 1 & 1 & 0 & 1 & 1 & 1 & 1 & 1 & 1 \\
\hline \multirow{8}{*}{$\begin{array}{l}\text { Pathway associated } \\
\text { with cell cycle, } \\
\text { proliferation, and } \\
\text { apoptosis }\end{array}$} & $\begin{array}{l}\text { Phosphoinositides and } \\
\text { their downstream targets }\end{array}$ & 1 & 0 & 0 & 1 & 0 & 1 & 0 & 1 & 1 & 0 & 0 & 0 & 0 \\
\hline & $\begin{array}{c}\text { Estrogen-responsive } \\
\text { protein Efp controls cell } \\
\text { cycle and breast tumors } \\
\text { growth }\end{array}$ & 1 & 1 & 1 & 1 & 1 & 1 & 0 & 0 & 1 & 0 & 0 & 0 & 0 \\
\hline & $\begin{array}{l}\text { Map kinase inactivation of } \\
\text { SMRT corepressor }\end{array}$ & 1 & 1 & 1 & 1 & 1 & 1 & 0 & 1 & 0 & 0 & 0 & 0 & 0 \\
\hline & $\begin{array}{c}\text { MAP Kinase Signaling } \\
\text { Pathway }\end{array}$ & 1 & 1 & 1 & 1 & 1 & 1 & 0 & 1 & 1 & 1 & 1 & 1 & 1 \\
\hline & $\begin{array}{c}\text { Roles of } \\
\beta \text {-arrestin-dependent } \\
\text { recruitment of Src kinases } \\
\text { in GPCR signaling }\end{array}$ & 1 & 1 & 1 & 1 & 1 & 1 & 0 & 1 & 1 & 1 & 1 & 1 & 1 \\
\hline & $\begin{array}{l}\text { Role of ERBB2 in signal } \\
\text { transduction and oncology }\end{array}$ & 1 & 1 & 1 & 1 & 1 & 1 & 0 & 1 & 1 & 1 & 1 & 1 & 1 \\
\hline & $\begin{array}{c}\text { Links between Pyk } 2 \text { and } \\
\text { MAP kinases }\end{array}$ & 1 & 1 & 1 & 1 & 1 & 1 & 0 & 1 & 1 & 1 & 1 & 1 & 1 \\
\hline & $\begin{array}{c}\text { NF- } \kappa \mathrm{B} \text { activation by } \\
\text { nontypeable Hemophilus } \\
\text { influenzae }\end{array}$ & 1 & 1 & 0 & 1 & 1 & 1 & 0 & 1 & 0 & 0 & 0 & 0 & 0 \\
\hline
\end{tabular}


TABLE 5: Continued.

\begin{tabular}{|c|c|c|c|c|c|c|c|c|c|c|c|c|c|c|}
\hline Category & Pathway & NXT & RA & RPR & RSM & RAS & RCX & SP & FC & CS & RAB & $\mathrm{RC}$ & $\mathrm{RM}$ & PT \\
\hline & $\begin{array}{c}\text { Influence of Ras and Rho } \\
\text { proteins on G1 to } S \\
\text { transition }\end{array}$ & 1 & 1 & 1 & 1 & 1 & 1 & 0 & 1 & 1 & 1 & 1 & 1 & 1 \\
\hline & Cell cycle: G1/S checkpoint & 1 & 1 & 1 & 1 & 1 & 1 & 0 & 0 & 1 & 0 & 0 & 0 & 0 \\
\hline & $\begin{array}{l}\text { Fc epsilon receptor I } \\
\text { signaling in mast cells }\end{array}$ & 1 & 1 & 1 & 1 & 1 & 1 & 0 & 1 & 1 & 1 & 1 & 1 & 1 \\
\hline & $\begin{array}{l}\text { Signaling of hepatocyte } \\
\text { growth factor receptor }\end{array}$ & 1 & 1 & 1 & 1 & 1 & 1 & 0 & 1 & 1 & 1 & 1 & 1 & 1 \\
\hline & Keratinocyte differentiation & 1 & 1 & 1 & 1 & 1 & 1 & 0 & 1 & 1 & 1 & 1 & 1 & 1 \\
\hline & $\begin{array}{c}\mathrm{RB} \text { tumor } \\
\text { Suppressor/checkpoint } \\
\text { signaling in response to } \\
\text { DNA damage }\end{array}$ & 1 & 1 & 1 & 1 & 1 & 1 & 0 & 0 & 1 & 0 & 0 & 0 & 0 \\
\hline & IL-3 Signaling Pathway & 1 & 1 & 1 & 1 & 1 & 1 & 0 & 1 & 1 & 1 & 1 & 1 & 1 \\
\hline & $\begin{array}{l}\text { Sprouty regulation of } \\
\text { tyrosine kinase signals }\end{array}$ & 1 & 1 & 1 & 1 & 1 & 1 & 0 & 1 & 1 & 1 & 1 & 1 & 1 \\
\hline \multirow{8}{*}{ Other pathways } & $\begin{array}{l}\text { Bioactive Peptide Induced } \\
\text { Signaling Pathway }\end{array}$ & 1 & 1 & 1 & 1 & 1 & 1 & 0 & 1 & 1 & 1 & 1 & 1 & 1 \\
\hline & amiPathway & 1 & 0 & 1 & 1 & 1 & 1 & 0 & 1 & 0 & 0 & 0 & 0 & 0 \\
\hline & Insulin Signaling Pathway & 1 & 1 & 1 & 1 & 1 & 1 & 0 & 1 & 1 & 1 & 1 & 1 & 1 \\
\hline & $\begin{array}{l}\text { p38 MAPK Signaling } \\
\text { Pathway }\end{array}$ & 1 & 1 & 1 & 1 & 1 & 1 & 0 & 1 & 1 & 1 & 1 & 1 & 1 \\
\hline & $\begin{array}{l}\text { The role of FYVE-finger } \\
\text { proteins in vesicle transport }\end{array}$ & 1 & 0 & 0 & 0 & 0 & 0 & 0 & 1 & 0 & 0 & 0 & 0 & 0 \\
\hline & $\begin{array}{l}\text { Mechanism of gene } \\
\text { regulation by peroxisome } \\
\text { proliferators via PPAR } \alpha\end{array}$ & 1 & 1 & 0 & 1 & 0 & 1 & 0 & 0 & 0 & 0 & 0 & 1 & 0 \\
\hline & $\begin{array}{l}\text { Phosphorylation of MEK1 } \\
\text { by cdk5/p35 downregulates } \\
\text { the MAP kinase pathway }\end{array}$ & 1 & 1 & 1 & 1 & 1 & 1 & 0 & 1 & 1 & 1 & 1 & 1 & 1 \\
\hline & $\begin{array}{c}\text { How progesterone initiates } \\
\text { oocyte membrane }\end{array}$ & 1 & 1 & 1 & 1 & 1 & 1 & 1 & 1 & 1 & 1 & 1 & 1 & 1 \\
\hline
\end{tabular}

Note. "1" means that the Chinese herbal medicine acts on the pathway while "0" means it does not. The pathways in each category are sorted by the significant differences in $P$ value.

Our study successfully predicted absorbable chemical compositions of NXT. These constituents primarily included ferulic acid, succinic acid, astragaloside IV, and tanshinone IIA. Ferulic acid, which is derived primarily from RA, RCX, RAS, and RAB, is reported to act as an angiogenic agent that augments angiogenesis, which is critical in ischemic diseases, such as myocardial infarction and stroke [8]. Succinic acid has been demonstrated to activate Akt phosphorylation to inhibit apoptosis and necrosis caused by cardiomyocyte hypoxia/reoxygenation [9]. Previous studies demonstrated that astragaloside IV could protect the heart through NOdependent mechanism [10]. NO has been confirmed to prevent the mitochondrial permeability transition pore from opening [11]. During early reperfusion, it can prevent the heart from reperfusion injury by inhibiting the opening of the mitochondrial permeability transition pore [12]. Tanshinone IIA also has cardioprotective effects, such as protection of cardiomyocytes from oxidative stress-triggered damage [13]. These reports were consistent with our results.
In addition to active ingredients, we also successfully predicted drug targets of NXT. The major targets were HRAS, MAP2K1, and MAPK14. The HRAS gene encodes the GTPase HRas, which is an enzyme known as transforming protein p21 [14]. With the ability to increase the effects of growth factor, HRas plays an important role in regulating the growth, differentiation, and death of endothelial cells [15]. The MAP2K1 gene encodes an enzyme named dual specificity mitogen-activated protein kinase kinase 1 , and MAPK14 encodes p38- $\alpha$. Both of these factors are closely related to inflammation and $\mathrm{p} 38-\alpha$ is also associated with cardiac hypertrophy via p38 MAPK activity in the heart. In addition, $\mathrm{p} 38-\alpha$ has been recognized as an isoenzyme of cardiovascular importance [16].

Among the numerous identified pathways, NFAT and hypertrophy of the heart (transcription in the broken heart) were ranked first. Nuclear factor of activated T-cells (NFAT) transcription factors, which have four different isoforms, plays crucial roles in the regulation of gene expression during 


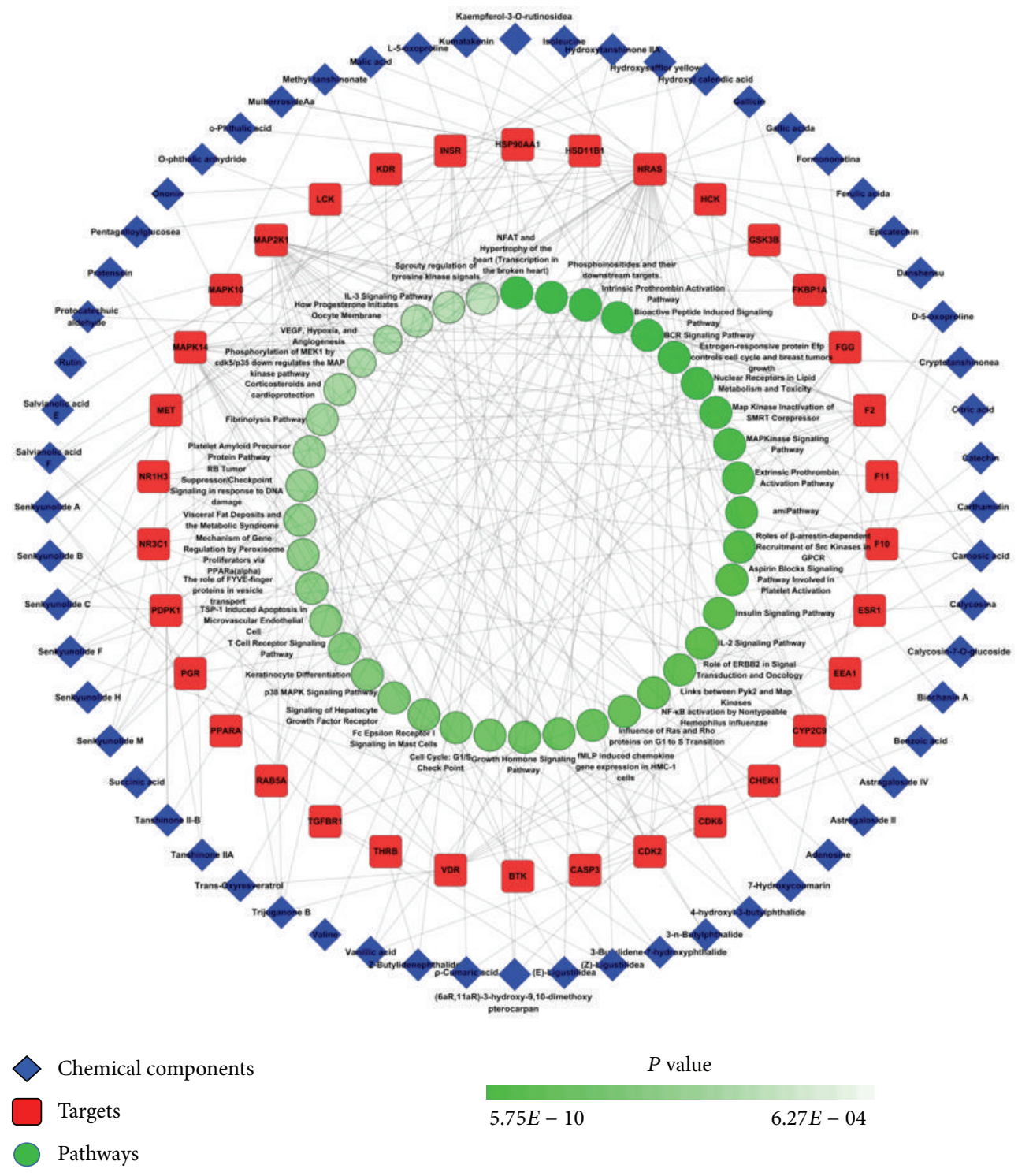

FIGURE 3: Pharmacology network of the "components-targets-pathways" regulated by NXT.

heart development [17]. The isoforms NFATc3 and NFATc4 are involved in hypertrophic development, while NFATc1 plays a key role in cardiac development [18]. The dephosphorylation of NFATs can promote calcineurin regulating immune response genes [19]. Via compensatory hypertrophy, the heart adapts to persistent stress conditions, but, over time, dysfunction and myocardial failure evolve [20]. Like NFAT and hypertrophy of the heart (transcription in the broken heart), most of these pathways are involved in the formation and regulation of cardiovascular disease, such as nuclear receptors in lipid metabolism and toxicity. Nuclear receptors include a superfamily of ligand-dependent transcription factors that regulate genetic networks that control cell growth, development, and metabolism. Regulating nuclear receptors is beneficial for patients with metabolic diseases, such as cardiovascular disease, due to the requirement for balance among a number of pathways for normal metabolic control [21]. These studies confirmed the validity of our study.

From the above results, we also found the different significances of the total of 16 herbs in NXT. According to Chinese Pharmacopoeia 2015, the content of RA in NXT is $66 \mathrm{~g}$, which is 2-3 times the content of any other herb in the whole prescription. It was reported that RA was the monarch drug of NXT and played a key role in improving the immune system, invigorating blood circulation, and the condition of myocardial ischemia and hypoxia [22]. Our study found that RA contained a lot of effective components, organic acids, and saponins and was critical source of the main active components of NXT. Through the comparison of the herbs involved in the top 40 pathways, RA was also proved to be the most important. In the top 40 pathways regulated by NXT, RA was involved in 33 pathways. Some other herbs, such as 


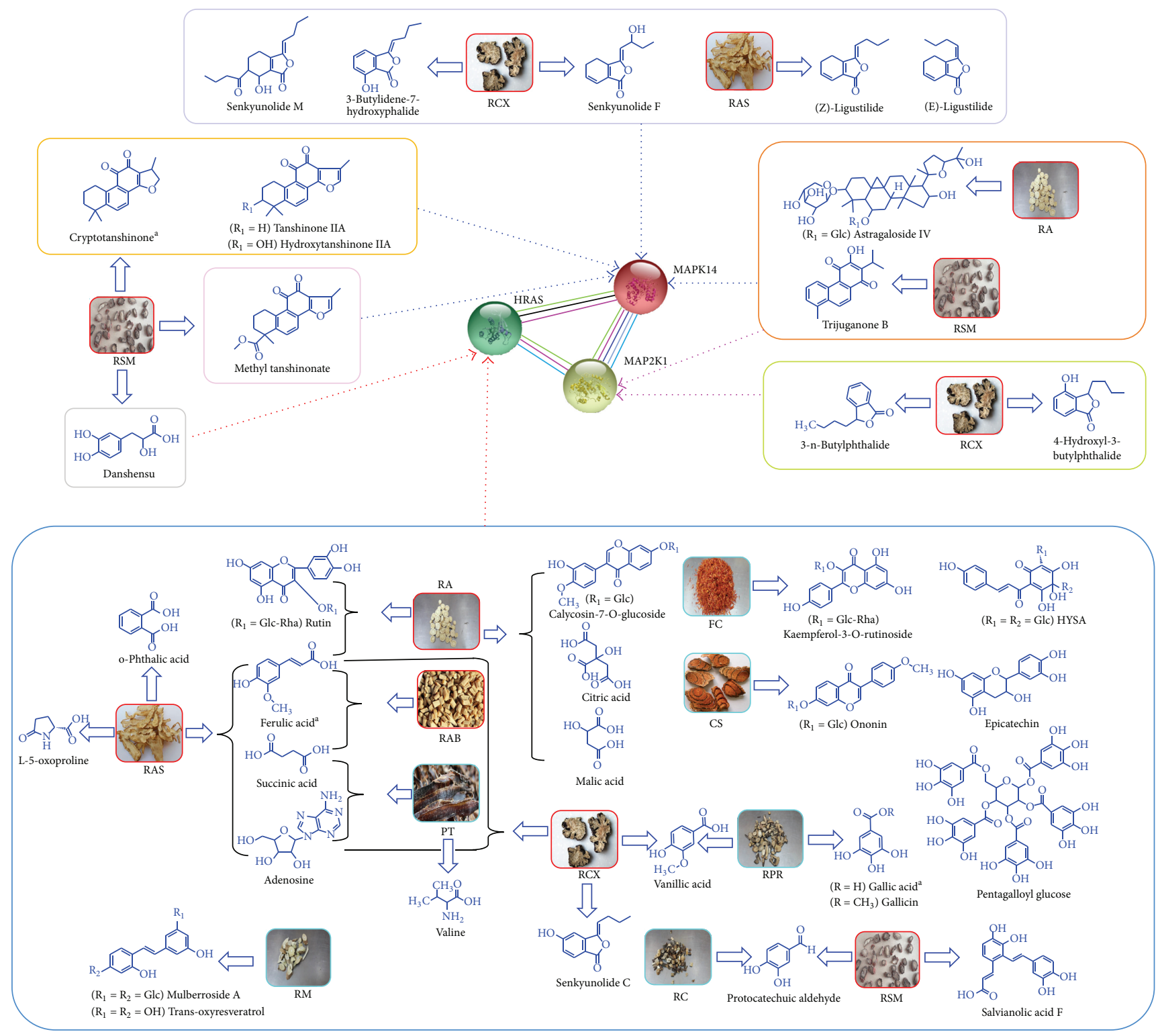

FIGURE 4: Network of major targets of NXT with corresponding compounds.

RSM, RCX, FC, and RAS, were also the important contents in the whole prescription of NXT.

The network pharmacology method used in this study is a novel methodology based on the construction of multilayer networks of disease phenotype-gene-drug to predict drug targets in a holistic manner and promote efficient drug discovery [23]. This method represents a breakthrough in comparison to the traditional herbal medicine research pattern "genetarget-disease" and initiates the new pattern "multiple genesmultiple targets-complex diseases" [24]. By this method, we proved that RA was the critical ingredient mainly involved in the regulation of metabolism and immunity in NXT. RAS was a major herb that regulated cell growth. RSM, RCX, and FC also played important roles in regulation of heart disease, blood vessels, and others. The results indicated that
NXT, a complex prescription in the treatment of complex diseases, played a therapeutic effect through multiple targets and multiple pathways. This was the first study to investigate the mechanism of NXT using this method, and we successfully predicted the main targets and pathways, providing a foundation for further research. This method has important value for the study of complex drugs and should be applied in future studies.

\section{Conclusion}

The main components that mediated the efficacy of NXT were organic acids, saponins, and tanshinones. Radix Astragali was the critical herbal medicine in NXT, which contained more active components than others and regulated more targets 
and pathways. NXT had a therapeutic effect on the treatment of heart diseases through the pattern "multiple componentsmultiple targets-multiple pathways."

\section{Abbreviations}

$\begin{array}{ll}\text { ACS: } & \text { Acute coronary syndrome } \\ \text { AMI: } & \text { Acute myocardial infarction } \\ \text { CBD: } & \text { Cerebrovascular disease } \\ \text { CVD: } & \text { Cardiovascular disease } \\ \text { ESI: } & \text { Electrospray ionization } \\ \text { HRAS: } & \text { Harvey rat sarcoma viral oncogene } \\ & \text { homolog } \\ \text { MAP2K1: } & \text { Mitogen-activated protein kinase } \\ & \text { kinase 1 } \\ \text { MAPK14: } & \text { Mitogen-activated protein kinase 14 } \\ \text { NFAT: } & \text { Nuclear factor of activated T cells } \\ \text { NXT: } & \text { Naoxintong capsule } \\ \text { TCMs: } & \text { Traditional Chinese medicines } \\ \text { UPLC/Q-TOF-MS: Ultraperformance liquid } & \text { chromatography/quadrupole } \\ & \text { time-of-flight mass spectrometry. }\end{array}$

\section{Competing Interests}

The authors have no conflicting financial interests.

\section{Authors' Contributions}

Xianghui Ma and Bin Lv contributed equally to this work.

\section{Acknowledgments}

This work was supported by the National Program for Key Basic Research Projects (2012CB518404), the NSFC (81202850 and 81125024), the Ministry of Education of PRC "Program for Innovative Research Team in University" (no. IRT1276), and the Program of International S\&T Cooperation Project of China (2015DFA30430).

\section{References}

[1] L. Su, Y. Li, B. Lv et al., "Clinical study on Naoxintong capsule for stroke recovery of Qi-deficiency and blood-stasis syndrome," Zhongguo Zhongyao Zazhi, vol. 36, no. 11, pp. 1530-1533, 2011.

[2] L.-X. Li, L. Chen, and H.-J. Zhao, "Effect of naoxintong capsule on the vascular endothelial function and the infarct size of patients with acute myocardial infarction," Chinese Journal of Integrated Traditional And Western Medicine, vol. 31, no. 12, pp. 1615-1618, 2011.

[3] S.-R. Li, T.-H. Wang, and B.-J. Zhang, "Effects of naoxintong capsule on the inflammation and prognosis in borderline lesion coronary heart disease patients," Chinese Journal of Integrated Traditional and Western Medicine, vol. 32, no. 5, pp. 607-611, 2012.
[4] J. Zhao, H. Zhu, S. Wang et al., "Naoxintong protects against atherosclerosis through lipid-lowering and inhibiting maturation of dendritic cells in LDL receptor knockout mice fed a highfat diet," Current Pharmaceutical Design, vol. 19, no. 33, pp. 58915896, 2013.

[5] X.-N. Zhong, H.-H. Wang, Z.-Q. Lu et al., "Effects of naoxintong on atherosclerosis and inducible nitric oxide synthase expression in atherosclerotic rabbit," Chinese Medical Journal, vol. 126, no. 6, pp. 1166-1170, 2013.

[6] F. Zhang, B. Huang, Y. Zhao et al., "BNC protects $\mathrm{H} 9 \mathrm{c} 2$ cardiomyoblasts from $\mathrm{H}_{2} \mathrm{O}_{2}$-induced oxidative injury through ERK1/2 signaling pathway," Evidence-based Complementary and Alternative Medicine, vol. 2013, Article ID 802784, 12 pages, 2013.

[7] J. Liu, Y. Liu, L. Wang et al., "The disease burden of cardiovascular and circulatory diseases in China, 1990 and 2010," Chinese Journal of Preventive Medicine, vol. 49, no. 4, pp. 315-320, 2015.

[8] C.-M. Lin, J.-H. Chiu, I.-H. Wu, B.-W. Wang, C.-M. Pan, and Y.H. Chen, "Ferulic acid augments angiogenesis via VEGF, PDGF and HIF-1 $\alpha$," Journal of Nutritional Biochemistry, vol. 21, no. 7, pp. 627-633, 2010.

[9] X.-L. Tang, J.-X. Liu, P. Li et al., "Protective effect of succinic acid on primary cardiomyocyte hypoxia/reoxygenation injury," China Journal of Chinese Materia Medica, vol. 38, no. 21, pp. 3742-3746, 2013.

[10] W.-D. Zhang, H. Chen, C. Zhang, R.-H. Liu, H.-L. Li, and H.-Z. Chen, "Astragaloside IV from Astragalus membranaceus shows cardioprotection during myocardial ischemia in vivo and in vitro," Planta Medica, vol. 72, no. 1, pp. 4-8, 2006.

[11] G. Wang, D. A. Liem, T. M. Vondriska et al., "Nitric oxide donors protect murine myocardium against infarction via modulation of mitochondrial permeability transition," American Journal of Physiology-Heart and Circulatory Physiology, vol. 288, no. 3, pp. H1290-H1295, 2005.

[12] D. J. Hausenloy, M. R. Duchen, and D. M. Yellon, "Inhibiting mitochondrial permeability transition pore opening at reperfusion protects against ischaemia-reperfusion injury," Cardiovascular Research, vol. 60, no. 3, pp. 617-625, 2003.

[13] J. Fu, H. Huang, J. Liu, R. Pi, J. Chen, and P. Liu, "Tanshinone IIA protects cardiac myocytes against oxidative stress-triggered damage and apoptosis," European Journal of Pharmacology, vol. 568, no. 1-3, pp. 213-221, 2007.

[14] M. W. Russell, D. J. Munroe, E. Bric et al., "A 500-kb physical map and contig from the Harvey ras-1 gene to the 1lp telomere," Genomics, vol. 35, no. 2, pp. 353-360, 1996.

[15] J. R. Burgoyne, D. J. Haeussler, V. Kumar et al., "Oxidation of HRas cysteine thiols by metabolic stress prevents palmitoylation in vivo and contributes to endothelial cell apoptosis," The FASEB Journal, vol. 26, no. 2, pp. 832-841, 2012.

[16] E. D. Martin, G. Felice De Nicola, and M. S. Marber, "New therapeutic targets in cardiology: P38 alpha mitogen-activated protein kinase for ischemic heart disease," Circulation, vol. 126, no. 3, pp. 357-368, 2012.

[17] E. van Rooij, P. A. Doevendans, C. C. De Theije, F. A. Babiker, J. D. Molkentin, and L. J. de Windt, "Requirement of nuclear factor of activated T-cells in calcineurin-mediated cardiomyocyte hypertrophy," The Journal of Biological Chemistry, vol. 277, no. 50, pp. 48617-48626, 2002.

[18] A. Rinne, N. Kapur, J. D. Molkentin et al., "Isoform- and tissuespecific regulation of the $\mathrm{Ca} 2+$-sensitive transcription factor NFAT in cardiac myocytes and heart failure," American Journal 
of Physiology-Heart and Circulatory Physiology, vol. 298, no. 6, pp. H2001-H2009, 2010.

[19] A. Rao, C. Luo, and P. G. Hogan, "Transcription factors of the NFAT family: regulation and function," Annual Review of Immunology, vol. 15, pp. 707-747, 1997.

[20] V. Papademetriou, "From hypertension to heart failure," Journal of Clinical Hypertension, vol. 6, no. 10, supplement 2, pp. 14-17, 2004.

[21] I. G. Schulman, "Nuclear receptors as drug targets for metabolic disease," Advanced Drug Delivery Reviews, vol. 62, no. 13, pp. 1307-1315, 2010.

[22] M.-W. Huang, H. Wang, W.-J. Zhong, X.-Y. Wu, and H. Chen, "Chinese herbal medicine Naoxintong capsule combined with dual antiplatelet therapy in a rat model of coronary microembolization induced by homologous microthrombi," Journal of Chinese Integrative Medicine, vol. 9, no. 1, pp. 38-48, 2011.

[23] Z.-H. Liu and X.-B. Sun, "Network pharmacology: new opportunity for the modernization of traditional Chinese medicine," Acta Pharmaceutica Sinica, vol. 47, no. 6, pp. 696-703, 2012.

[24] A. L. Hopkins, "Network pharmacology: the next paradigm in drug discovery," Nature Chemical Biology, vol. 4, no. 11, pp. 682690, 2008. 


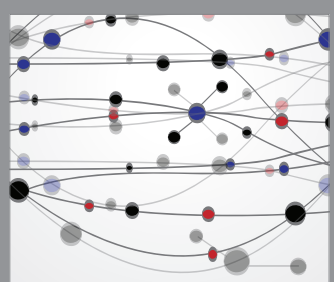

The Scientific World Journal
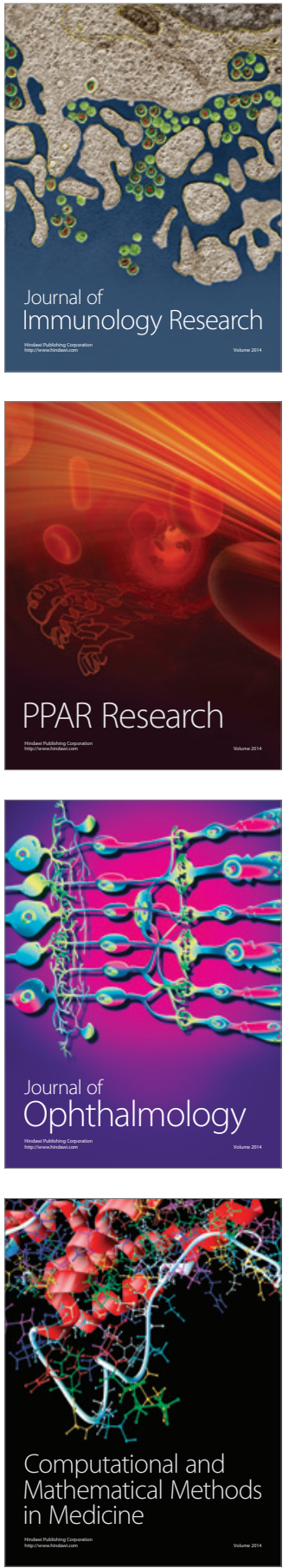

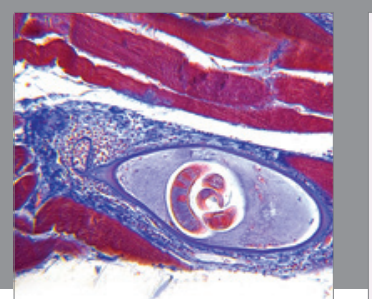

Gastroenterology Research and Practice

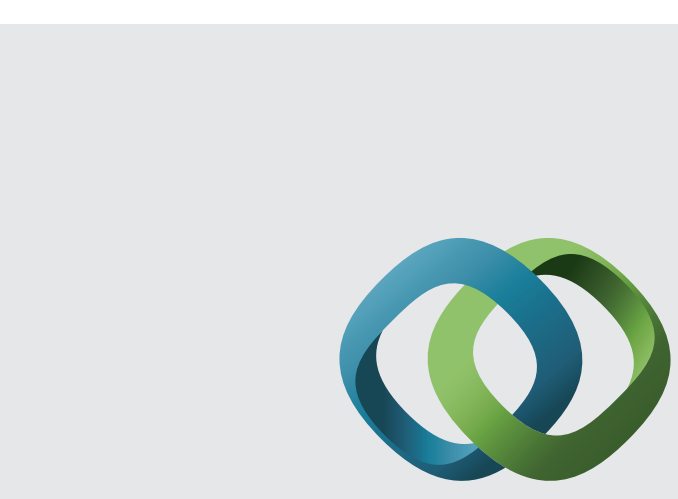

\section{Hindawi}

Submit your manuscripts at

http://www.hindawi.com
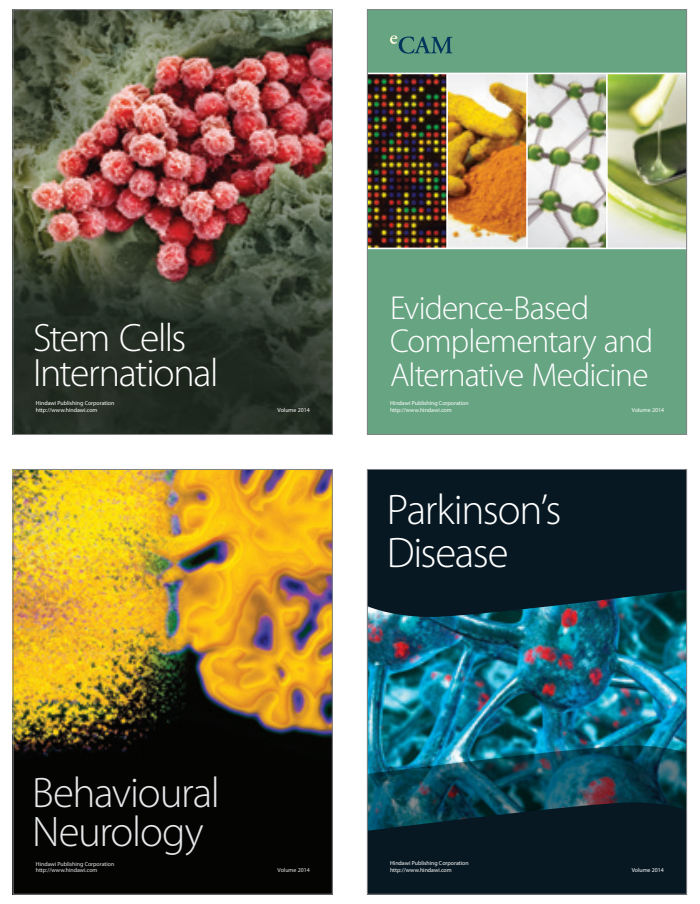
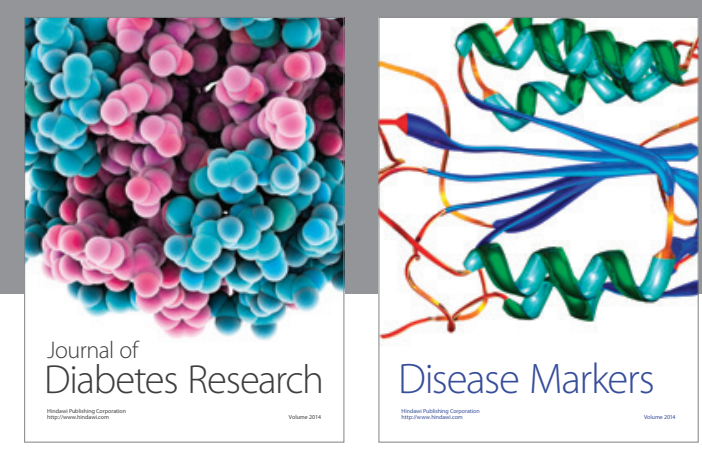

Disease Markers
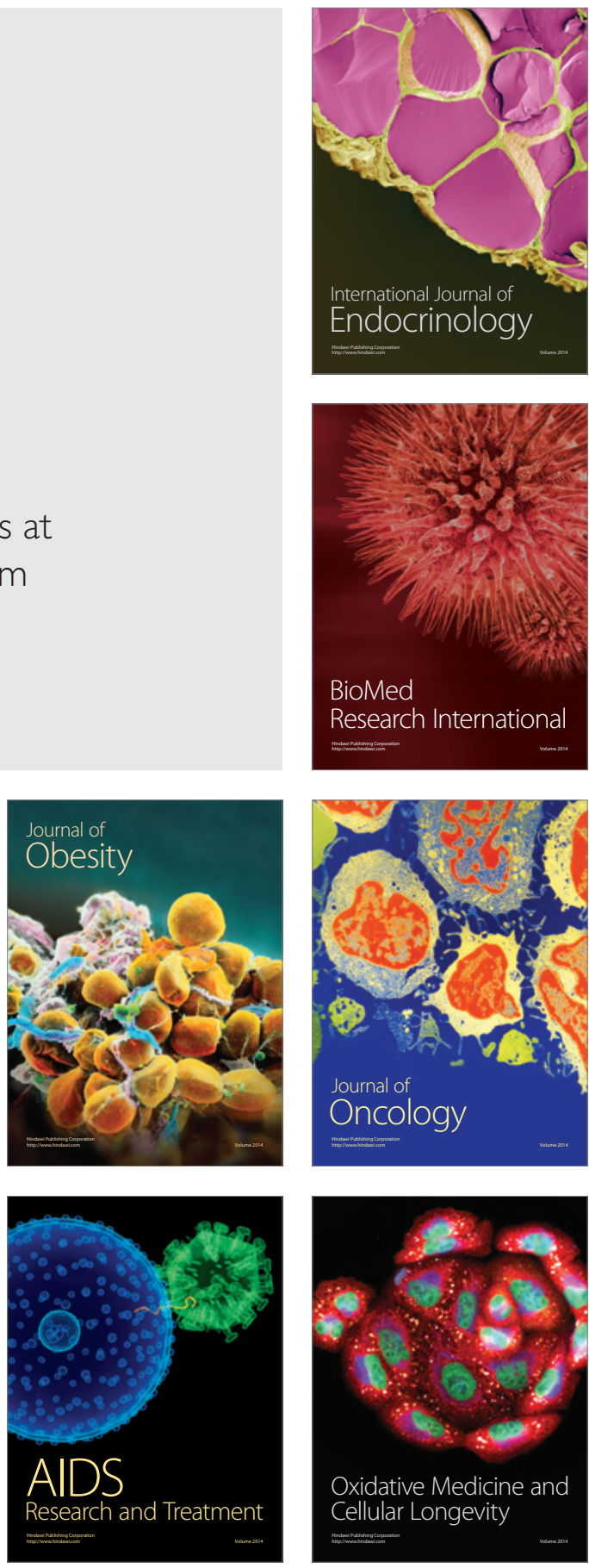